\title{
Systematic Review \\ Systematic Review on the Association of Radiomics with Tumor Biological Endpoints
}

\author{
Agustina La Greca Saint-Esteven ${ }^{1, *(\mathbb{D}}$, Diem Vuong ${ }^{1} \mathbb{D}$, Fabienne Tschanz ${ }^{2}$, Janita E. van Timmeren ${ }^{1}$,

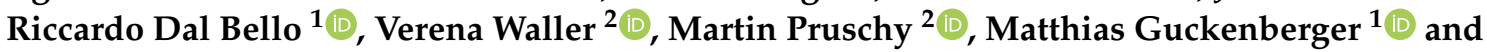 \\ Stephanie Tanadini-Lang ${ }^{1}$ (D)
}

1 Department of Radiation Oncology, University Hospital Zurich and University of Zurich, 8091 Zurich, Switzerland; diem.vuong@usz.ch (D.V.); Janita.vanTimmeren@usz.ch (J.E.v.T.); riccardo.dalbello@usz.ch (R.D.B.); matthias.guckenberger@usz.ch (M.G.); stephanie.tanadini-lang@usz.ch (S.T.-L.)

2 Laboratory of Applied Radiobiology, Department of Radiation Oncology, University of Zurich, 8091 Zurich, Switzerland; fabienne.tschanz@uzh.ch (F.T.); verena.waller@uzh.ch (V.W.); martin.pruschy@uzh.ch (M.P.)

* Correspondence: agustina.lagreca@usz.ch

check for updates

Citation: La Greca Saint-Esteven, A.; Vuong, D.; Tschanz, F.; van Timmeren, J.E.; Dal Bello, R.; Waller, V.; Pruschy,

M.; Guckenberger, M.; Tanadini-Lang, S. Systematic Review on the Association of Radiomics with Tumor Biological Endpoints. Cancers 2021, 13, 3015. https://doi.org/10.3390/ cancers13123015

Academic Editor: Claudio Fiorino

Received: 19 May 2021

Accepted: 11 June 2021

Published: 16 June 2021

Publisher's Note: MDPI stays neutral with regard to jurisdictional claims in published maps and institutional affiliations.

Copyright: (c) 2021 by the authors. Licensee MDPI, Basel, Switzerland. This article is an open access article distributed under the terms and conditions of the Creative Commons Attribution (CC BY) license (https:// creativecommons.org/licenses/by/ $4.0 /)$.
Simple Summary: In this systematic review, we aim to highlight existing literature devoted to the study of an association between medical imaging radiomics and cancer biological endpoints. The use of radiomics as an ancillary tool in cancer treatment would allow for a non-invasive, inexpensive, three-dimensional characterization of the tumor phenotype, contributing to the delivery of precision medicine. Nonetheless, its clinical application remains a challenge, as extensive, multi-center validation studies of radiomic features connection with tumor biology are required. In this review, we performed a search in PubMed database for peer-reviewed studies which evaluate the association between radiomic features and the following set of clinically relevant tumor markers: anaplastic lymphoma kinase (ALK), v-raf murine sarcoma viral oncogene homolog B1 (BRAF), epidermal growth factor (EGFR), human epidermal growth factor receptor 2 (HER-2), isocitrate dehydrogenase (IDH), antigen Ki-67, kirsten rat sarcoma viral oncogene homolog (KRAS), programmed cell death ligand 1 (PD-L1), tumor protein p53 (TP-53) and vascular endothelial growth factor (VEGF).

Abstract: Radiomics supposes an alternative non-invasive tumor characterization tool, which has experienced increased interest with the advent of more powerful computers and more sophisticated machine learning algorithms. Nonetheless, the incorporation of radiomics in cancer clinical-decision support systems still necessitates a thorough analysis of its relationship with tumor biology. Herein, we present a systematic review focusing on the clinical evidence of radiomics as a surrogate method for tumor molecular profile characterization. An extensive literature review was conducted in PubMed, including papers on radiomics and a selected set of clinically relevant and commonly used tumor molecular markers. We summarized our findings based on different cancer entities, additionally evaluating the effect of different modalities for the prediction of biomarkers at each tumor site. Results suggest the existence of an association between the studied biomarkers and radiomics from different modalities and different tumor sites, even though a larger number of multi-center studies are required to further validate the reported outcomes.

Keywords: radiomics; tumor biology; cancer; imaging biomarker; tumor molecular marker

\section{Introduction}

Cancer precision medicine involves therapy adaptation to improve clinical outcome based on patient-specific characteristics as well as the tumor-specific molecular profile. The advent of high-throughput gene-sequencing techniques in the last decade has allowed for the identification of multiple tumor molecular markers, also known as signature molecules, 
which correspond to genomic changes that affect gene and protein expression [1,2]. These encompass a great variety of biological molecules such as nucleic acids, proteins, peptides, lipid metabolites and other small molecules, and their assessment can be beneficial for diagnosis, prognosis, or prediction of therapy response. Besides assisting in clinical-decision processes, these signature molecules may also hold the potential for new personalized molecular targeted or immunologic therapies.

On the other hand, the fast-evolving field of radiomics has experienced an increased interest in the past decade, especially within cancer research, due to accumulating evidence of an association between quantitative medical imaging features and clinical and biological endpoints [3]. The underlying principle behind radiomics is that medical images enclose latent information which can be unveiled through the extraction of radiomic features, i.e., quantitative features which describe the shape, size, intensity and texture of a region of interest. The most common imaging modalities used for this purpose are computed tomography (CT), magnetic resonance imaging (MRI), positron emission tomography (PET) and ultrasound (US). Recent advances in machine learning and computer hardware, together with the availability of large-scale medical imaging data, have redefined radiomics as a powerful tool for precision medicine in clinical-decision support systems [4]. Moreover, the non-invasiveness nature of radiomics supposes a great advantage when compared to current gold-standard techniques for tumor phenotype characterization.

The purpose of this systematic review was to determine which radiomic features have been linked to tumor biology in peer-reviewed studies and, thus, could be potentially incorporated in cancer precision medicine. To this end, we chose to summarize current findings assessing the potential association of radiomics with ten classic proteins or genes which are relevant clinical prognostic markers, and which may be targeted by either small molecular inhibitors or antibodies. These are: anaplastic lymphoma kinase (ALK), v-raf murine sarcoma viral oncogene homolog B1 (BRAF), epidermal growth factor (EGFR), human epidermal growth factor receptor 2 (HER-2), isocitrate dehydrogenase (IDH), antigen Ki-67, kirsten rat sarcoma viral oncogene homolog (KRAS), programmed cell death ligand 1 (PD-L1), tumor protein p53 (TP-53) and vascular endothelial growth factor (VEGF).

These factors, when mutated or over-expressed, play important roles in cancer progression and growth (EGFR, HER-2, KRAS, BRAF, ALK), angiogenesis (VEGF), cell cycle regulation and cell death (TP-53), the immune response (PD-L1), and metabolic regulation (IDH). At the same time, it is important to understand the proliferation kinetics (Ki-67 proliferation marker) of the respective cancer types as that can also influence the treatment response.

- EGFR, HER-2 and ALK are all receptor tyrosine kinases, located on the cell surface and activated through the binding of ligands, mostly growth factors. This leads to the activation of a whole range of downstream signaling cascades and results in cell survival, proliferation and migration $[5,6]$.

- $\quad$ KRAS and BRAF are the genes responsible for making the proteins K-ras and B-raf, which are, amongst others, involved in important signaling pathways (e.g., Ras-RafMAPK, PI3-K-AKT) [7,8]. Mutation and down-/up-regulation of any of those kinases can lead to malignancy and especially cancer formation.

- VEGF is a signaling factor promoting the formation of new blood vessels. To grow and metastasize, solid cancers require blood supply, which they attain by expressing VEGF to form supporting vasculature [9].

- $\quad \mathrm{TP}-53$ is involved in the regulation and progression through the cell cycle; monitors genomic stability and can induce apoptosis. It is one of the most prominent tumorsuppressors [10].

- $\quad$ PD-L1 is involved in suppressing the adaptive arm of the immune system. By upregulating PD-L1 expression, cancer cells may evade the host immune system [11].

- IDH catalyzes the decarboxylation of isocitrate. Through this metabolic deregulation, cancer progression can be initiated or supported [12]. 
- $\mathrm{Ki}-67$ is a protein that is present during all active phases of the cell cycle but absent in resting (quiescent) cells [13]. Therefore, this cellular proliferation marker is frequently used to distinguish fast growing cell populations, such has cancer cells, from normal cells.

Throughout this review, the term "biomarker" refers, for the sake of simplicity, to any of the above-mentioned biological endpoints. This is also in accordance with the World Health Organization (WHO), which defines biomarker as "any substance, structure, or process that can be measured in the body, or its products and influences or predicts the incidence of outcome or disease" [14].

\section{Materials and Methods}

The analysis was conducted according to the PRISMA-P Preferred Reporting Items for Systematic Reviews and Meta-Analyses statement [15]. The protocol for this systematic review was registered at PROSPERO (CRD42020207220) and is available at https: / / www. crd.york.ac.uk/prospero/display_record.php?ID=CRD42020207220 (accessed on 11 June 2021). No amendments were performed with respect to the published protocol.

\subsection{Literature Search}

The search was conducted in PubMed database. According to PRISMA guidelines, article selection was carried out via multiple steps. The literature search was performed using the query "Radiomics [All Fields] AND keyword [All Fields]", where keyword corresponded to one of the ten molecular markers under study (i.e., ALK, BRAF, EGFR, HER-2, IDH, Ki-67, KRAS, TP-53, PD-L1 and VEGF) and the possible variations in its naming (e.g., HER2 and HER-2). The full list of queries is provided in the Supplementary Materials (List S1). In total, twenty independent searches were performed. No records were included from other sources such as direct correspondence with authors. The search had no start date limit and was concluded on 31 March 2020.

For each independent search, all retrieved studies were collected, and duplicates were posteriorly removed using the open-source reference management software Zotero [16].

\subsection{Eligibility Criteria}

During the first screening phase, those studies which did not fulfil the following requirements were excluded: (1) the article had to be written in English, (2) the study had to be a scientific article excluding reviews, (3) the topic had to be related to biomarkers in cancer. Following this step, every article was assigned to one of the following categories, depending on the cancer site: breast, central nervous system (CNS), gastrointestinal, liver, lung and others.

The full-text articles were then assessed for eligibility. An article was excluded from the final analysis if at least one of the following criteria applied: (1) only one of the two groups, biomarker-negative or biomarker-positive, patients were included in the study, (2) the total number of patients was less than 40, (3) the association between the biomarker and radiomics features was not investigated, (4) the biomarker analyzed was not among the ten biomarkers defined in the search and (5) less than 20 image features were investigated.

\subsection{Analysis}

Those articles that satisfied the screening and eligibility criteria were included in the following analysis, with each tumor site corresponding to a dedicated subsection in this review. First, the distribution of the number of patients included within all the studies was evaluated. The frequency of investigation of a given biomarker for each tumor site was collected in a dedicated table, together with the total number of studies on each tumor site and on each biomarker.

For each study, we gathered the following information when available: the studied biological endpoint and its alteration, e.g., mutation on a specific exon, over-expression, etc.; the imaging modality; the origin of the dataset; the training set size; the validation set 
size and type of validation, i.e., internal, temporally independent, external, leave-one-out-, 3-, 5- and 10-fold cross-validation (LOOCV, 3-CV, 5-CV, 10-CV) or bootstrap methods; the initial number of studied radiomic features; the application of feature reduction and feature robustness analysis methods; the reported performance, i.e., the area under the receiver-operating characteristic curve (AUC), classification accuracy or c-index; the public availability of the code and/or data; the reported quality score of the radiomics study, e.g., the transparent reporting of a multivariable prediction modelling for individual prognosis or diagnosis (TRIPOD) score [17] or Radiomics Quality Score (RQS) [4].

Furthermore, radiomic features of the best performing models on the training set were identified for each combination of tumor site, biomarker and image modality, in order to provide, when possible, a visual interpretation of the findings. For consistency, performance on the training set was evaluated since external validation was only performed on a small fraction of the studies. Moreover, in this comparison, the selection was limited to models based solely on radiomic features, i.e., mixed models including clinical-radiological data were excluded. This process was done independently by each of the authors in the systematic review. If the study provided a visual interpretation of such features, it was recorded. Otherwise, whenever possible, the missing interpretation was provided by the authors.

In accordance with PRISMA guidelines, a strategy for bias risk minimization was adopted as follows: the processes of screening, eligibility evaluation and extraction of data for the meta-analysis were performed independently by authors ALG, DV, FT, RDB and VW. Each author analyzed one specific tumor site. The more experienced authors JEvT, ST-L, MG and MP supervised the process and guaranteed a uniform and unbiased analysis throughout the different tumor sites. A detailed description of this process can be found on the PRISMA checklist in the Supplementary Materials (List S2).

\section{Results}

\subsection{Literature Search, Eligibility Criteria and Study Selection}

A diagram summarizing the study selection workflow following PRISMA guidelines is shown in Figure 1. A total of 304 records were first retrieved from PubMed. After duplicate removal, 183 articles were left for screening. The first screening excluded 33 articles, leaving 150 full-text studies for the eligibility assessment. After further evaluation, 46 references were excluded because they did not meet the conditions previously defined. As a result, 104 articles were included in the current review.

The size of the dataset under study varied significantly among the reported papers (43-1010 patients). As above-mentioned, studies including less than 40 patients in total were excluded from the analysis during the screening phase. The mean number of patients included was 198. The distribution is shown in Figure 2.

The frequency of investigation of a given biomarker with respect to each tumor site is presented in Table 1. It should be noted that multiple keywords, i.e., multiple biomarkers, were allowed for the same article. Therefore, the total sum of the entries $(n=125)$ is greater than the number of full-text papers included in the systematic review $(n=104)$. Similarly, those articles that investigated more than one tumor site were included in each of the corresponding subsections. The most frequently studied entity was lung cancer, followed by CNS tumors and breast cancer. The most frequently analyzed biomarker was EGFR, followed by Ki-67 and IDH. The association between EGFR and radiomics in lung cancer was the most frequently investigated $(n=26)$. 

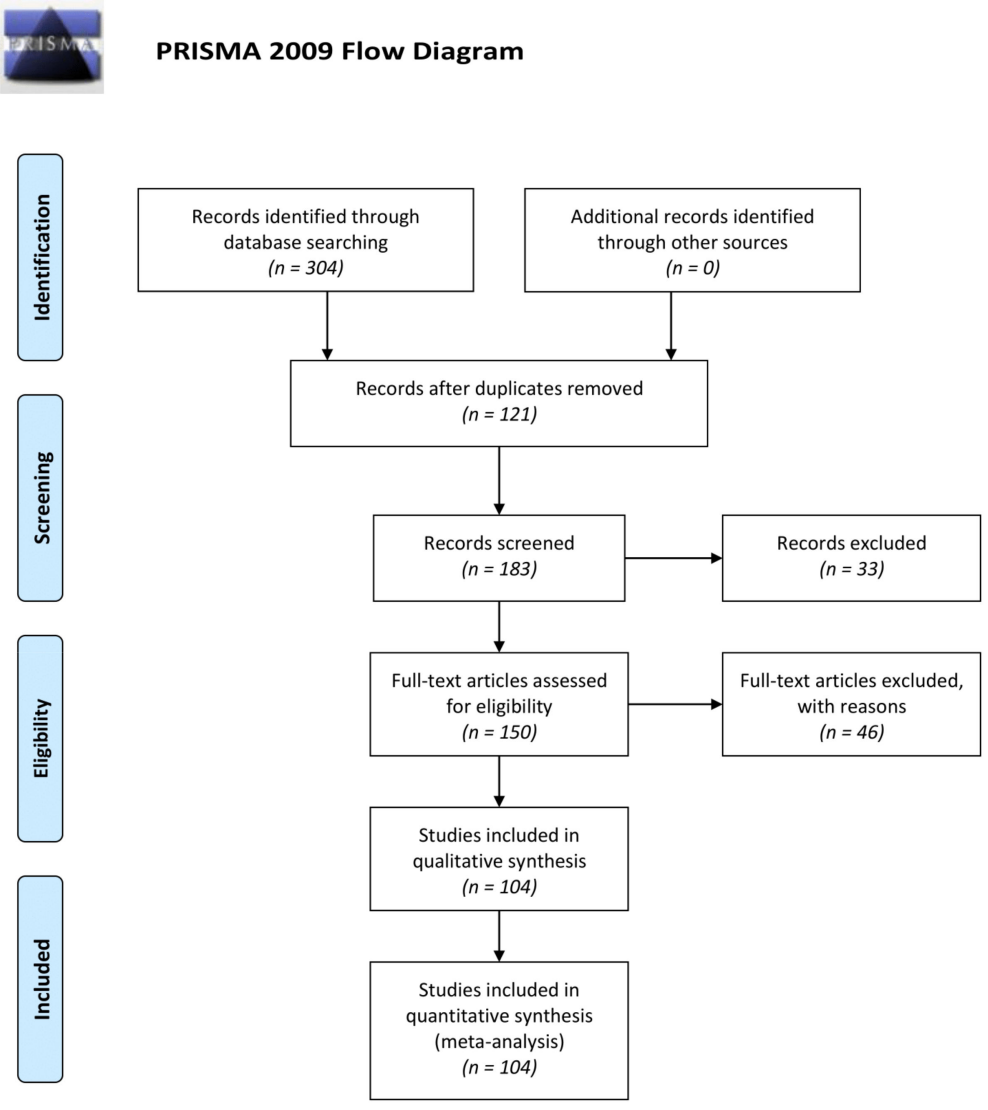

From: Moher D, Liberati A, Tetzlaff J, Altman DG, The PRISMA Group (2009). Preferred Reporting fems for Systematic Reviews and MetaAnalyses: The PRISMA Statement. PLoS Med 6(7): e1000097. doi:10.1371/journal.pmed1000097

For more information, visit www.prisma-statement.org.

Figure 1. Flow diagram of the study selection process according to PRISMA guidelines [15].

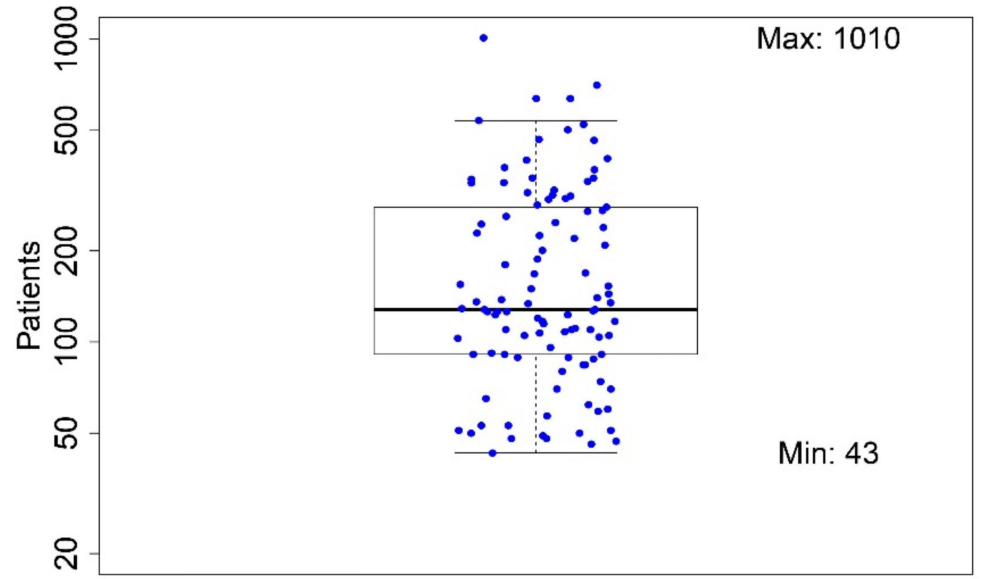

Figure 2. Distribution of the number of patients in the studies included in the analysis. 
Table 1. Frequency of investigation of each biomarker and entity site.

\begin{tabular}{cccccccc}
\hline & Breast & CNS & GI & Liver & Lung & Others & Total \\
\hline ALK & 0 & 0 & 0 & 0 & 3 & 0 & 3 \\
BRAF & 0 & 0 & 1 & 0 & 0 & 2 & 3 \\
EGFR & 0 & 5 & 0 & 0 & 26 & 1 & 32 \\
HER-2 & 10 & 0 & 2 & 0 & 0 & 0 & 12 \\
IDH & 0 & 24 & 0 & 0 & 0 & 0 & 24 \\
Ki-67 & 8 & 5 & 3 & 3 & 2 & 2 & 23 \\
KRAS & 0 & 0 & 7 & 0 & 5 & 0 & 12 \\
PD-L1 & 0 & 0 & 0 & 2 & 3 & 1 & 6 \\
TP-53 & 1 & 2 & 2 & 0 & 1 & 1 & 7 \\
VEGF & 0 & 1 & 0 & 1 & 0 & 1 & 3 \\
\hline TOTAL & 19 & 37 & 15 & 6 & 40 & 8 & 125 \\
\hline
\end{tabular}

\section{2. $C N S$}

\subsubsection{Summary}

A total of thirty-six studies were found which associated CNS tumor molecular markers with clinical imaging radiomic features, using conventional magnetic resonance imaging (MRI) alone $(n=21)$ [18-38], advanced MRI sequences $(n=11)$ [39-49], Positron Emission Tomography (PET, $n=3$ ) [50-52] or Amide Proton Transfer-weighted (APTw, $n=1$ ) imaging [53]. Glioma was the tumor type analyzed in the vast majority $(n=35)[18-34,36-53]$, including lower-grade glioma (LGG) $(n=20)[18,21-30,32,34,36,37,46-49,53]$, gliomas of all grades $(n=9)[33,38,42-45,50-52]$, and glioblastoma (GBM) [17,18,29,37-39]. One study focused on pituitary macroadenoma [35]. IDH genotype was the most frequently studied biomarker $(n=24)$ [18-30,38,42,43,45-50,52,53], followed by EGFR $(n=5)[31,32,39-41]$, Ki-67 $(n=5)$ [33-35,44,51], TP-53 $(n=2)[30,36]$ and VEGF $(n=1)$ [37]. All studies showed a significant association between the biomarker and radiomic features (AUC $=0.70-0.99$ ). Thirty-four studies validated their models either on internal cohorts, temporally independent cohorts or through cross-validation/bootstrap methods. Two studies validated their models using externally acquired datasets. Three studies were prospective registered studies $[21,47,53]$. None of the studies reported any radiomics quality measure. The findings of this section are summarized in Table 2 . 
Table 2. An overview of the radiomic studies included in the CNS cancer section.

\begin{tabular}{|c|c|c|c|c|c|c|c|c|c|c|c|c|}
\hline Study & Biomarker & r Alteration & Modality & ity Dataset Origin & Training & Validation & Feature Reduction & $\begin{array}{c}\text { Feature } \\
\text { Robustness }\end{array}$ & $\begin{array}{l}\text { \# Radiomic } \\
\text { Features }\end{array}$ & Additional Features & $\begin{array}{c}\text { Predictive Power } \\
\text { Measure = Mean (95\% } \\
\text { Confidence Interval) }\end{array}$ & $\begin{array}{l}\text { Open } \\
\text { Source }\end{array}$ \\
\hline Akbari et al. [41] & EGFR & $\begin{array}{l}\text { Variant III } \\
\text { mutation } \\
\text { (deletion of } \\
\text { exons 2-7) }\end{array}$ & $\begin{array}{l}\text { MRI, } \\
\text { DWI, } \\
\text { PWI }\end{array}$ & $\begin{array}{l}\text { Hospital of the } \\
\text { University of } \\
\text { Pennsylvania, } \\
\text { Philadelphia, US } \\
\text { Osaka International }\end{array}$ & 75 & $54^{*}$ & no & no & 421 & $\begin{array}{c}16 \text { tumor spatial } \\
\text { location features; } \\
\text { peritumoral } \\
\text { heterogeneity index }\end{array}$ & $\begin{array}{c}\mathrm{AUC}=0.92 \\
\text { Accuracy }=88.9 \%\end{array}$ & Code \\
\hline Arita et al. [26] & IDH & $\begin{array}{l}\text { Isoforms } 1 \\
\text { (codon 132) and } 2 \\
\text { (codon 172) } \\
\text { mutations }\end{array}$ & MRI & $\begin{array}{c}\text { Cancer Institute, } \\
\text { Osaka, Japan; } \\
\text { National Cancer } \\
\text { Center Research } \\
\text { Institute, } \\
\text { Tokyo, Japan }\end{array}$ & 111 & $58^{*}$ & yes & no & 50 & $\begin{array}{l}59 \text { tumor spatial } \\
\text { location features }\end{array}$ & Accuracy $=87 \%$ & $\begin{array}{l}\text { Code } \\
\text { Features }\end{array}$ \\
\hline Binder et al. [39] & EGFR & $\begin{array}{c}\text { Extracellular } \\
\text { A289D/T/V, } \\
\text { R108G/K and } \\
\text { G598V mutations }\end{array}$ & $\begin{array}{l}\text { MRI, } \\
\text { PWI, } \\
\text { DWI }\end{array}$ & $\begin{array}{l}\text { Hospital of the } \\
\text { University of } \\
\text { Pennsylvania, } \\
\text { Philadelphia, US }\end{array}$ & 260 & - & yes & no & 2088 & $\begin{array}{l}11 \text { tumor spatial } \\
\text { location features; } \\
5 \text { glioma diffusion } \\
\text { properties from tumor } \\
\text { biophysical models }\end{array}$ & $\begin{array}{l}\text { Significant correlation } \\
(p<0.0444)\end{array}$ & Code \\
\hline Choi et al. [20] & IDH & $\begin{array}{l}\text { Isoforms } 1 \\
\text { (codon 132) } \\
\text { mutation }\end{array}$ & MRI & $\begin{array}{l}\text { TCIA/TCGA-GBM; } \\
\text { St. Mary's Hospital, } \\
\text { Seoul, South Korea } \\
\text { Osaka International }\end{array}$ & 45 & $91 * *$ & yes & no & 107 & - & $\begin{array}{c}\text { AUC }=0.904(0.805,1.0) \\
\text { Accuracy }=86.8 \%(63.7 \\
97.8)\end{array}$ & $\begin{array}{c}\text { Images and } \\
\text { ROI } \\
\text { partially }\end{array}$ \\
\hline $\begin{array}{l}\text { Fukuma et al. } \\
\text { [27] }\end{array}$ & IDH & $\begin{array}{l}\text { Isoforms } 1 \\
\text { (codon 132) and } 2 \\
\text { (codon 172) } \\
\text { mutations }\end{array}$ & MRI & $\begin{array}{c}\text { Cancer Institute, } \\
\text { Osaka, Japan; } \\
\text { National Cancer } \\
\text { Center Research } \\
\text { Institute, } \\
\text { Tokyo, Japan }\end{array}$ & 127 & $10-\mathrm{CV}$ & yes & no & 61 & $\begin{array}{c}3 \text { tumor spatial } \\
\text { location features; } \\
\text { 4000 DL features; age }\end{array}$ & Accuracy $=73.1 \%$ & - \\
\hline Han et al. [53] & IDH & $\begin{array}{l}\text { Isoforms } 1 \\
\text { (codon 132) } \\
\text { mutation }\end{array}$ & $\mathrm{APTw}$ & $\begin{array}{l}\text { Tangdu Hospital, } \\
\text { Xian, China }\end{array}$ & 49 & $10 *$ & yes & yes & 1044 & - & $\begin{array}{c}\text { AUC }=0.952 \\
\text { Accuracy }=0.892\end{array}$ & $\begin{array}{c}\text { Images on } \\
\text { request }\end{array}$ \\
\hline Kim et al. [47] & $\mathrm{IDH}$ & $\begin{array}{l}\text { Isoforms } 1 \\
\text { (codon 132) } \\
\text { mutation }\end{array}$ & $\begin{array}{l}\text { MRI, } \\
\text { DWI, } \\
\text { PWI }\end{array}$ & $\begin{array}{l}\text { Asan Medical Center, } \\
\text { Seoul, South Korea }\end{array}$ & 127 & $28^{* * *}$ & yes & yes & 6472 & - & $\begin{array}{c}\text { AUC }=0.747(0.66-0.83) \\
\text { Accuracy }=65.3 \%\end{array}$ & - \\
\hline Kong et al. [51] & Ki-67 & $\begin{array}{l}\text { High Ki-67 } \\
\text { expression as } \\
>10 \%\end{array}$ & $\begin{array}{l}\text { FDG- } \\
\text { PET }\end{array}$ & $\begin{array}{l}\text { Peking Union Medical } \\
\text { College Hospital, } \\
\text { Beijing, China }\end{array}$ & 82 & $41^{*}$ & yes & no & 1561 & $\begin{array}{l}\text { Age; sex; metabolic } \\
\text { pattern; SUVmax; } \\
\text { SUVmean }\end{array}$ & $\begin{array}{c}\mathrm{AUC}=0.73 \\
\text { Accuracy }=78 \%\end{array}$ & - \\
\hline $\begin{array}{l}\text { Kuthuru et al. } \\
{[28]}\end{array}$ & IDH & $\begin{array}{l}\text { Isoforms } 1 \\
\text { (codon 132) } \\
\text { mutation }\end{array}$ & MRI & TCGA/TCIA-LGG & 108 & $10-\mathrm{CV}$ & no & no & No & $\begin{array}{c}>35,000 \text { histogram of } \\
\text { oriented gradients, } \\
\text { scale-invariant feature } \\
\text { transform and voxel } \\
\text { intensities }\end{array}$ & $\begin{array}{l}\text { AUC }=0.8224 \\
(0.7856-0.8575)\end{array}$ & $\begin{array}{l}\text { Images and } \\
\text { ROI }\end{array}$ \\
\hline Lee et al. [31] & EGFR & mutation & MRI & TCGA/TCIA-GBM & 44 & $3-\mathrm{CV}$ & no & no & - & $\begin{array}{l}36 \text { spatial diversity } \\
\text { features }\end{array}$ & $\begin{array}{c}\text { AUC }=0.845 \\
\text { Accuracy }=0.79\end{array}$ & $\begin{array}{l}\text { Images and } \\
\text { ROI }\end{array}$ \\
\hline Lee et al. [48] & IDH & $\begin{array}{l}\text { Isoforms } 1 \\
\text { (codon 132) } \\
\text { mutation }\end{array}$ & $\begin{array}{l}\text { MRI, } \\
\text { DWI, } \\
\text { PWI }\end{array}$ & $\begin{array}{l}\text { Samsung Medical } \\
\text { Center, Seoul, } \\
\text { South Korea }\end{array}$ & 88 & $35^{* * *}$ & yes & no & 82 & - & Accuracy $=83.4 \%$ & - \\
\hline
\end{tabular}


Table 2. Cont

\begin{tabular}{|c|c|c|c|c|c|c|c|c|c|c|c|c|}
\hline Study & Biomarker & er Alteration & Modality & ity Dataset Origin & Training & Validation & Feature Reduction & $\begin{array}{c}\text { Feature } \\
\text { Robustness }\end{array}$ & $\begin{array}{l}\text { \# Radiomic } \\
\text { Features }\end{array}$ & Additional Features & $\begin{array}{c}\text { Predictive Power } \\
\text { Measure = Mean (95\% } \\
\text { Confidence Interval) }\end{array}$ & $\begin{array}{l}\text { Open } \\
\text { Source }\end{array}$ \\
\hline Li et al. [50] & IDH & $\begin{array}{c}\text { Isoforms } 1 \\
\text { (codon 132) and } 2 \\
\text { (codon 172) } \\
\text { mutations }\end{array}$ & $\begin{array}{l}\text { FDG- } \\
\text { PET }\end{array}$ & $\begin{array}{l}\text { Peking Union Medical } \\
\text { College Hospital, } \\
\text { Beijing, China }\end{array}$ & 84 & $43^{*}$ & yes & no & 1561 & $\begin{array}{l}\text { Age; sex; metabolic } \\
\text { pattern; SUVmax; } \\
\text { SUVmean }\end{array}$ & $\begin{array}{l}\text { AUC }=0.900 \\
(0.877-0.923)\end{array}$ & Code \\
\hline Li et al. [19] & (c & $\begin{array}{l}\text { Isoforms } 1 \\
\text { (codon 132) and } 2 \\
\text { (codon 172) } \\
\text { mutations }\end{array}$ & A & $\begin{array}{c}\text { TCGA/TCIA-GBM; } \\
\text { Sun Yat-sen } \\
\text { University Cancer } \\
\text { Center, Guangzhou, } \\
\text { China; The 3rd } \\
\text { Affiliated Hospital of } \\
\text { Sun Yat-sen } \\
\text { University, } \\
\text { Guangzhou, China; } \\
\text { Guangzhou General } \\
\text { Hospital of } \\
\text { Guangzhou Military } \\
\text { Command, } \\
\text { Guangzhou, China }\end{array}$ & 118 & $107^{* *}$ & yes & no & 1614 & Sex; age; KPS & $\begin{array}{c}\mathrm{AUC}=0.96 \\
\text { Accuracy }=97 \%\end{array}$ & $\begin{array}{c}\text { Images and } \\
\text { ROI } \\
\text { (partially) }\end{array}$ \\
\hline Li et al. [33] & Ki-67 & $\begin{array}{c}\text { High Ki-67 } \\
\text { expression as > } \\
25 \%\end{array}$ & MRI L & $\begin{array}{l}\text { The Second Hospital } \\
\text { of Hebei Medical } \\
\text { University, Tangshan, } \\
\text { Hebei, China }\end{array}$ & 50 & $\begin{array}{l}3-\mathrm{CV}, \\
5-\mathrm{CV}, \\
\text { boot- } \\
\text { strap }\end{array}$ & yes & no & 396 & - & $\begin{array}{c}\text { AUC }=0.713 \\
(0.568-0.832) \\
\text { Accuracy }=66.0 \%\end{array}$ & - \\
\hline Li et al. [32] & EGFR & $\begin{array}{c}\text { High EGFR } \\
\text { expression as > } \\
30 \%\end{array}$ & MRI & $\begin{array}{l}\text { Beijing Tiantan } \\
\text { Hospital, Beijing, } \\
\text { China } \\
\text { Chinese Glioma }\end{array}$ & 200 & $70^{*}$ & yes & no & 431 & - & $\begin{array}{c}\mathrm{AUC}=0.95 \\
\text { Accuracy }=90.0 \%\end{array}$ & \\
\hline Li et al. [36] & TP-53 & mutation & MRI G & $\begin{array}{l}\text { Genome Atlas, Beijing } \\
\text { Tiantan Hospital, } \\
\text { Beijing, China }\end{array}$ & 180 & $92 *$ & yes & no & 431 & - & $\begin{array}{c}\text { AUC }=0.763 \\
\text { Accuracy }=70.7 \%\end{array}$ & $\begin{array}{l}\text { Images and } \\
\text { ROI }\end{array}$ \\
\hline Li et al. [34] & Ki-67 & $\begin{array}{c}\text { High Ki-67 } \\
\text { expression as }> \\
10 \%\end{array}$ & MRI & $\begin{array}{c}\text { Beijing Tiantan } \\
\text { Hospital, Beijing, } \\
\text { China; Chinese } \\
\text { Glioma Genome Atlas }\end{array}$ & 78 & $39 *$ & yes & no & 431 & - & $\begin{array}{c}\mathrm{AUC}=0.90 \\
\text { Accuracy }=88.6 \%\end{array}$ & - \\
\hline Li et al. [22] & IDH & $\begin{array}{l}\text { Isoforms } 1 \\
\text { (codon 132) } \\
\text { mutation }\end{array}$ & MRI & $\begin{array}{l}\text { Huashan Hospital, } \\
\text { Shangai, China }\end{array}$ & 229 & LOOCV & yes & no & 671 & 16,384 DLR features & $\begin{array}{c}\text { AUC }=0.9521 \\
\text { Accuracy }=92.44 \%\end{array}$ & - \\
\hline Liu et al. [21] & IDH & $\begin{array}{l}\text { Isoforms } 1 \\
\text { (codon 132) } \\
\text { mutation }\end{array}$ & MRI & $\begin{array}{l}\text { Beijing Tiantan } \\
\text { Hospital, Beijing, } \\
\text { China; }\end{array}$ & 158 & $102 * * *$ & yes & yes & 431 & - & $\mathrm{AUC}=0.99$ & - \\
\hline $\begin{array}{l}\text { Lohmann et al. } \\
\text { [52] }\end{array}$ & IDH & $\begin{array}{l}\text { Isoforms } 1 \\
\text { (codon 132) } \\
\text { mutation }\end{array}$ & $\begin{array}{l}\text { FET- } \\
\text { PET }\end{array}$ & $\begin{array}{l}\text { University Hospital } \\
\text { RWTH Aachen }\end{array}$ & 84 & $\begin{array}{l}5-\mathrm{CV}, \\
10-\mathrm{CV}\end{array}$ & no & yes & 33 & $\begin{array}{c}\text { Slope; TTP; } \\
\text { mean tumor-to-brain } \\
\text { ratio; maximum } \\
\text { tumor-to-brain ratio }\end{array}$ & $\begin{array}{c}\mathrm{AUC}=0.79 \\
\text { Accuracy }=80.0 \%\end{array}$ & - \\
\hline Lu et al. [43] & IDH & mutation & $\begin{array}{l}\text { MRI, } \\
\text { DWI }\end{array}$ & $\begin{array}{c}\text { TCGA/TCIA-LGG; } \\
\text { TCGA/TCIA-GBM; } \\
\text { TCIA-REMBRANDT; } \\
\text { Taipei Medical } \\
\text { University, Taipei, } \\
\text { Taiwan }\end{array}$ & 214 & $70 * *$ & yes & no & 39,212 & - & Accuracy $=88.9-91.7 \%$ & $\begin{array}{c}\text { Images and } \\
\text { ROI } \\
\text { (partially) }\end{array}$ \\
\hline
\end{tabular}


Table 2. Cont

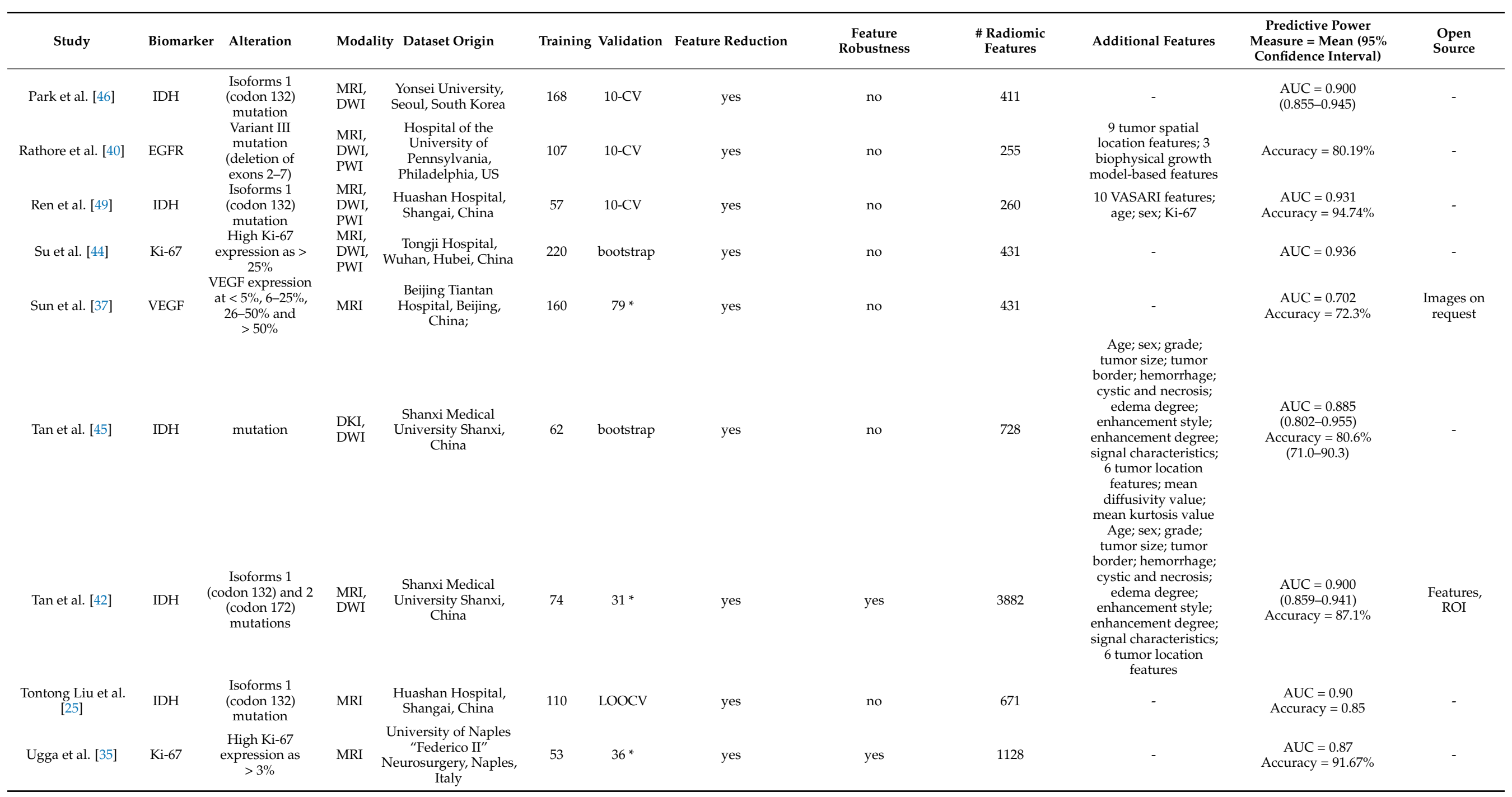


Table 2. Cont.

\begin{tabular}{|c|c|c|c|c|c|c|c|c|c|c|c|c|}
\hline Study & Biomarker & Alteration & Modality & ty Dataset Origin & Training & Validation & Feature Reduction & $\begin{array}{c}\text { Feature } \\
\text { Robustness }\end{array}$ & $\begin{array}{l}\text { \# Radiomic } \\
\text { Features }\end{array}$ & Additional Features & $\begin{array}{c}\text { Predictive Power } \\
\text { Measure = Mean (95\% } \\
\text { Confidence Interval) }\end{array}$ & $\begin{array}{l}\text { Open } \\
\text { Source }\end{array}$ \\
\hline Wu et al. [38] & IDH & $\begin{array}{l}\text { Isoforms 1 } \\
\text { (codon 132) } \\
\text { mutation }\end{array}$ & MRI & $\begin{array}{l}\text { TCGA/TCIA-LGG; } \\
\text { TCGA/TCIA-GBM }\end{array}$ & 126 & bootstrap & yes & no & 698 & $\begin{array}{l}6 \text { tumor growth } \\
\text { model parameters }\end{array}$ & $\begin{array}{c}\text { AUC }=0.931 \\
\text { Accuracy }=0.885\end{array}$ & Images, ROI \\
\hline Wu et al. [18] & IDH & $\begin{array}{l}\text { Isoforms 1 } \\
\text { (codon 132) } \\
\text { mutation }\end{array}$ & MRI & $\begin{array}{l}\text { Huashan Hospital, } \\
\text { Shangai, China }\end{array}$ & 80 & $25^{*}$ & yes & no & - & $\begin{array}{l}968 \text { dictionary } \\
\text { features }\end{array}$ & Accuracy $=88.0 \%$ & - \\
\hline Yu et al. [24] & IDH & $\begin{array}{l}\text { Isoforms 1 } \\
\text { (codon 132) } \\
\text { mutation }\end{array}$ & MRI & $\begin{array}{l}\text { Huashan Hospital, } \\
\text { Shangai, China }\end{array}$ & 92 & LOOCV & no & no & - & $\begin{array}{l}116 \text { tumor spatial } \\
\text { location features }\end{array}$ & $\begin{array}{c}\mathrm{AUC}=0.71 \\
\text { Accuracy }=72.0 \%\end{array}$ & - \\
\hline Zhang et al. [30] & $\begin{array}{l}\text { IDH, } \\
\text { TP-53 }\end{array}$ & mutation & MRI & TCGA/TCIA-LGG & 73 & $30 *$ & yes & yes & 260 & 16 VASARI features & $\begin{array}{c}\text { IDH: } \text { AUC }=0.792 \\
\text { Accuracy }=80.0 \% \\
\text { TP-53: AUC }=0.869 \\
\text { Accuracy }=85.0 \%\end{array}$ & Images, ROI \\
\hline Zhou et al. [29] & IDH & mutation & MRI & TCGA/TCIA-LGG & 84 & bootstrap & yes & no & 3360 & $\begin{array}{c}30 \text { VASARI features; } \\
\text { age; sex; KPS; } \\
\text { histological type; } \\
\text { grade; laterality; } \\
\text { location }\end{array}$ & $\mathrm{AUC}=0.86$ & $\begin{array}{l}\text { Images, ROI, } \\
\text { code }\end{array}$ \\
\hline
\end{tabular}

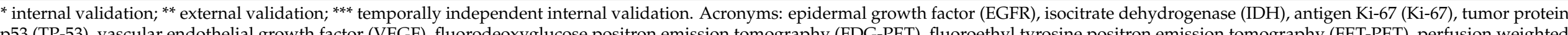

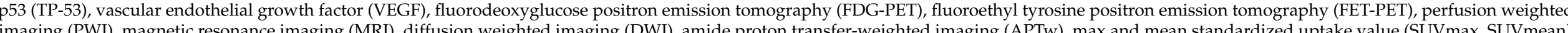

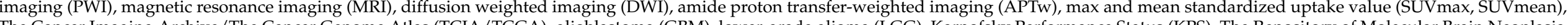

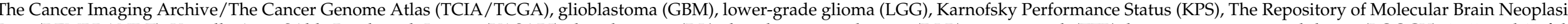

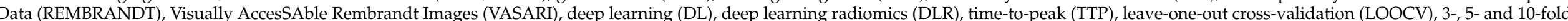
cross-validation (3-, 5- and 10-CV), area under the curve (AUC). 


\subsubsection{IDH}

A total of fifteen studies were found which investigated the power of MR radiomics to predict IDH genotype in glioma (AUC $=0.70-0.99$, accuracy $=73.1-97 \%$ ) $[18-30,38]$. Four studies exclusively employed MR radiomics $[20,21,23,25]$, whereas eight studies used a combination of traditional radiomic features with other types of imaging features such as location parameters $(n=5)$ [23-27], Visually AcceSAble Rembrandt Images (VASARI) features $(n=3)[19,29,30]$, deep learning radiomics (DLR, $n=2)[22,27]$, and tumor growth model parameters $(n=1)$ [38]. Three studies based their models completely on non-conventional radiomics: one of them employed purely anatomical location features [24], another one used histogram of oriented gradients (HoGs), raw voxel intensities and scale-invariant feature transform (SIFT) descriptors [28] and the third one used fine texture features obtained by k-means singular value decomposition (K-SVD), a dictionary learning algorithm [18]. Three of the fifteen studies also incorporated clinical-radiological parameters into their models $[19,27,29]$.

The best predictive performance was achieved by Li et al. on MR images of glioblastoma patients by means of a random forests (RF) model based on gray-level co-occurrence matrix (GLCM), grey-level run-length matrix (GLRLM) and grey-level size zone matrix (GLSZM) textural features together with patient age (AUC $=0.96$, external validation) [19]. Among MR radiomics in both, lower- and higher-grade gliomas, relevant features for IDH mutation status prediction were associated with textural heterogeneity, suggesting that IDH wild-type tumors are more heterogeneous and more structurally complex than IDH-mutant ones $[19,20,26]$. Another feature that was significantly linked with IDH mutation status was tumor mean surface-to-volume ratio, which was lower in IDH-mutant cases [20,23]. Moreover, IDH-mutant gliomas were found to occur more frequently in the frontal, insular and temporal lobes $[24,26]$.

A total of seven studies were found which combined MR radiomics with diffusion weighted imaging (DWI), perfusion weighted imaging (PWI) and/or diffusion kinetic imaging (DKI) features to predict IDH mutation status in glioma patients (AUC $=0.747-$ 0.931) [42,43,45-49]. Among these studies, three of them incorporated clinical-radiological parameters in their modelling $[42,43,45]$ and one employed additional VASARI imaging features [49]. The best performance on an external cohort was achieved by Lu et al., who proposed two support vector machine (SVM) models based on MR and DWI features together with patient age and sex to predict IDH mutation status in GBM and LGG patients separately (accuracy $=88.9-91.7 \%$, external validation) [43]. Similar to MRI radiomics, DWI and PWI textural and intensity features describing increased tumor heterogeneity were associated with IDH wild-type tumors. Moreover, IDH wild-type LGGs were found to have smaller minimum Apparent Diffusion Coefficient (ADC) and Cerebral Blood Volume (CBV) values, which could indicate an increased tumor proliferation index and increased malignancy $[47,49]$.

Two studies were found which used PET radiomics in conjunction with clinicalradiological parameters to predict IDH status in gliomas. One of them used fluoroethyltyrosine (FET)-PET standard parameter slope and one texture feature (accuracy $=80.0 \%$, 10-CV) [52], while the other one combined fluorodeoxyglucose (FDG)-PET radiomics with age and tumor metabolism to achieve an AUC of 0.90 on an internal validation set [50]. Among FDG-PET radiomics, the feature sphericity was found to play a significant role in IDH mutation status prediction, indicating that IDH-mutant gliomas are less spherical than IDH wild-type in FDG-PET scans. Lastly, one study used APTw radiomics to predict IDH status in LGG patients (AUC $=0.84$, internal validation) [53]. GLCM and GLRLM radiomic features describing tumor heterogeneity were identified as main contributors of IDH genotype prediction, with IDH-mutant tumors being more homogeneous.

\subsubsection{EGFR}

In total, two studies were found which investigated the relationship between MR radiomics and EGFR alterations in glioma, more precisely, between EGFR over-expression in 
LGG patients and EGFR mutation in GBM patients [31,36]. The former proposed a logistic regression model based on 41 radiomics features (AUC $=0.95$, internal validation) [36], and the latter study employed a symbolic regression method based on non-conventional MR spatial diversity descriptors (AUC $=0.845$, cross-validation) [31]. In both cases, MR features describing tumor textural heterogeneity and shape irregularity were linked to EGFR, suggesting increased diversity in EGFR-mutated and EGFR-amplified tumors.

Three studies were found which employed MR radiomics together with DWI and PWI radiomics to predict EGFR mutation status in GBM patients. Binder et al. studied a variety of EGFR missense mutations and concluded that EGFR mutation at alanine 289 (EGFRA289D/T/V) presented a unique radiographic phenotype. Authors reported significantly lower average T1 signal, higher relative cerebral blood volume and longer major axis in EGFR-A289D/T/V-mutant tumors among other features [39]. The two remaining studies investigated the prediction of EGFR mutation at exons 2-7 (EGFRvIII) and incorporated additional imaging features on their modelling such as location parameters, tumor growth model parameters and the peritumoral heterogeneity index. Authors reported predictive accuracies of $73.58 \%$ [40] and $87 \%$ [41] on a temporally independent and on an internal validation cohort, respectively. Authors of the three above-mentioned studies suggested that EGFR-mutant tumors present an increase in shape variability and water concentration as well as a decreased cell density.

\subsubsection{Ki-67}

Three studies were found which investigated the association between Ki-67 expression and MR radiomics in CNS tumors: one in lower grade glioma (AUC $=0.9$, accuracy $=88.6 \%$, internal validation) [34], one in both, lower and higher-grade gliomas (AUC $=0.713$, accuracy $=66 \%$, cross-validation) [33] and one in pituitary macroadenoma (accuracy $=96.7 \%$, internal validation) [35]. In the three studies, Ki-67 expression was associated with increased textural heterogeneity. One study was found which combined MR, DWI and PWI radiomics to predict Ki-67 expression in grade I-IV gliomas (AUC $=0.936$, training cohort) [44]. Authors reported that DWI features were more strongly associated with Ki-67 than the other two imaging modalities. Another study focused on Ki-67 prediction using FDG-PET radiomics (AUC $=0.76$, internal validation) [51].

Among the five studies, different cut-off thresholds were employed to distinguish between low- and high-expressing tumors, with two studies using $10 \%$ [34,51], two studies using $25 \%$ [34,44] and one study using 3\% [35].

\subsubsection{TP-53}

Two studies evaluated the power of MR radiomics for TP-53 mutation status prediction in LGGs with a varying performance (AUC $=0.763-0.869$, internal validation) $[30,36]$. One of them also included VASARI imaging features in the modelling. Authors concluded that TP-53 mutant gliomas are more heterogeneous and present higher water content.

\subsubsection{VEGF}

One study was found which investigated the use of conventional MR radiomics to predict VEGF expression in LGGs (AUC $=0.702$, internal validation) [37].

\subsection{Breast Cancer}

\subsubsection{Summary}

Sixteen studies which assessed the relationship between breast tumor molecular markers and radiomic features were identified. The imaging modalities studied were PWI $(n=9)$ [54-62], conventional MRI $(n=2)$ [63,64], DWI $(n=1)$ [65], DWI+PWI [66], Digital Breast Tomography (DBT, $n=1)$ [67], PET/CT $(n=1)$ [68] and standard 2D digital mammography (DMG, $n=1$ ) [69]. Most studies focused on invasive breast cancer $(n=13)[54-60,63-67,69]$, while three studies investigated non-invasive breast cancer types $[61,62,68]$. HER-2 was the most frequently investigated biomarker $(n=10)$, and 
all studies showed some degree of linkage between HER-2 status and radiomic features (AUC $=0.65-0.95)[54,56-60,62,64,68,69]$. Eight studies examined Ki-67, and all except one study found a significant association to radiomics features (AUC $=0.70-0.81)[56,57,62,63,65-68]$. One study investigated TP-53 status $(n=1)(\mathrm{AUC}=0.88)$ [55]. Thirteen studies employed internal validation sets or cross-validation and bootstrap methods datasets. None of the studies reported any radiomics quality measure and only two of them were registered prospective studies [64,67]. The findings of this section are summarized in Table 3. 
Table 3. An overview of the radiomic studies included in the breast cancer section.

\begin{tabular}{|c|c|c|c|c|c|c|c|c|c|c|c|c|}
\hline Study & Biomarker & Alteration & Modality & Dataset Origin & Training & Validation & $\begin{array}{c}\text { Feature } \\
\text { Reduction }\end{array}$ & $\begin{array}{c}\text { Feature } \\
\text { Robustness }\end{array}$ & $\begin{array}{l}\text { \# Radiomic } \\
\text { Features }\end{array}$ & $\begin{array}{l}\text { Additional } \\
\text { Features }\end{array}$ & $\begin{array}{c}\text { Predictive Power } \\
\text { Measure = Mean (95\% } \\
\text { Confidence Interval) }\end{array}$ & Open Source \\
\hline $\begin{array}{c}\text { Antunovic et al. } \\
\text { [68] }\end{array}$ & $\begin{array}{l}\text { HER-2, } \\
\text { Ki-67 }\end{array}$ & $\begin{array}{c}\text { HER-2: } \\
\text { positive (IHC } \\
3+\text { ) vs. } \\
\text { negative (IHC } \\
0 \text { or 1) } \\
\text { Ki-67: High } \\
\text { expression at } \\
>20 \%\end{array}$ & $\begin{array}{c}\text { FDG- } \\
\text { PET/CT }\end{array}$ & $\begin{array}{l}\text { Humanitas } \\
\text { Hospital, Milan, } \\
\text { Italy }\end{array}$ & 43 & - & yes & no & 20 & $\begin{array}{l}\text { MTV, SUV } \text { mean } \\
\text { and TLG }\end{array}$ & $\begin{array}{l}\text { HER-2: Significant } \\
\text { correlation }(p= \\
0.021-0.046) \\
\text { Ki-67: No significant } \\
\text { correlation }\end{array}$ & - \\
\hline Braman et al. [61] & HER-2 & mutation & PWI & $\begin{array}{c}\text { Cleveland Medical } \\
\text { Center, Cleveland, } \\
\text { Ohio, US; City of } \\
\text { Hope } \\
\text { Comprehensive } \\
\text { Cancer Center, } \\
\text { Duarte, California, } \\
\text { US; Yale Cancer } \\
\text { Center, New Haven, } \\
\text { Connecticut, US; } \\
\text { Brown University } \\
\text { Onnology Research } \\
\text { Group, Providence, } \\
\text { Rhode Island, US; } \\
\text { TCIA/TCGA- } \\
\text { BRCA }\end{array}$ & 117 & $3-\mathrm{CV}$ & yes & no & 495 & - & AUC $=0.71(0.63-0.79)$ & images \\
\hline Fan et al. [59] & HER-2 & $\begin{array}{c}\text { positive (IHC } \\
3+\text { ) vs. } \\
\text { negative (IHC } \\
0 \text { or 1) }\end{array}$ & PWI & $\begin{array}{l}\text { Zhejiang Cancer } \\
\text { Hospital, } \\
\text { Hangzhou, China }\end{array}$ & 60 & $36^{*}$ & yes & no & 65 & $\begin{array}{l}\text { status; } 29 \text { dynamic } \\
\text { features from BPE } \\
\text { and the lesion; } \\
9 \text { bilateral } \\
\text { differences in BPE }\end{array}$ & $\mathrm{AUC}=0.947$ & - \\
\hline $\begin{array}{l}\text { Leithner et al. } \\
\text { [64] }\end{array}$ & HER-2 & mutation & PWI, DWI & $\begin{array}{l}\text { First Affiliated } \\
\text { Hospital of } \\
\text { Zhejiang Chinese } \\
\text { Medical University, } \\
\text { Hangzhou, China } \\
\text { Memorial Sloan } \\
\text { Kettering Cancer } \\
\text { Center, New York, } \\
\text { USA; Medical } \\
\text { University Vienna, } \\
\text { Vienna, Austria }\end{array}$ & 144 & LOOCV & yes & no & 352 & - & Accuracy $=73.6 \%$ & Code \\
\hline
\end{tabular}


Table 3. Cont.

\begin{tabular}{|c|c|c|c|c|c|c|c|c|c|c|c|c|}
\hline Study & Biomarker & Alteration & Modality & Dataset Origin & Training & Validation & $\begin{array}{c}\text { Feature } \\
\text { Reduction }\end{array}$ & $\begin{array}{c}\text { Feature } \\
\text { Robustness }\end{array}$ & $\begin{array}{l}\text { \# Radiomic } \\
\text { Features }\end{array}$ & $\begin{array}{l}\text { Additional } \\
\text { Features }\end{array}$ & $\begin{array}{c}\text { Predictive Power } \\
\text { Measure = Mean (95\% } \\
\text { Confidence Interval) }\end{array}$ & Open Source \\
\hline Li et al. [54] & HER-2 & mutation & PWI & $\begin{array}{l}\text { TCGA/TCIA- } \\
\text { BRCA }\end{array}$ & 91 & LOOCV & yes & no & 24 & $\begin{array}{c}10 \text { kinetic features } \\
\text { (maximum contrast } \\
\text { enhancement, TTP, } \\
\text { uptake rate, } \\
\text { washout rate, curve } \\
\text { shape index, } \\
\text { enhancement at } \\
\text { first post-contrast, } \\
\text { SER, volume of } \\
\text { most enhancing } \\
\text { voxels, total rate } \\
\text { variation, } \\
\text { normalized total } \\
\text { rate variation) and } \\
4 \text { enhancement- } \\
\text { variance kinetic } \\
\text { features (maximum } \\
\text { variance of } \\
\text { enhancement, TTP, } \\
\text { variance increase } \\
\text { rate, and variance } \\
\text { decrease rate) }\end{array}$ & $\mathrm{AUC}=0.65$ & images \\
\hline Li et al. [58] & HER-2 & mutation & PWI & $\begin{array}{l}\text { Cancer Hospital of } \\
\text { Liaoning, China }\end{array}$ & 637 & LOOCV & yes & no & 137 & $\begin{array}{l}5 \text { kinetic features } \\
\text { (standard deviation, } \\
\text { mean, maximum } \\
\text { value, enhancement } \\
\text { rate, absorption } \\
\text { rate) }\end{array}$ & $\begin{array}{c}\mathrm{AUC}=0.83 \\
\text { Accuracy }=87.0 \%\end{array}$ & - \\
\hline Liang et al. [63] & Ki-67 & $\begin{array}{l}\text { High } \\
\text { expression at } \\
>14 \%\end{array}$ & MRI & $\begin{array}{c}\text { Guangdong } \\
\text { General Hospital, } \\
\text { Guangdong } \\
\text { Academy of } \\
\text { Medical Sciences, } \\
\text { Guangzhou, China } \\
\text { \& Southern Medical } \\
\text { University, } \\
\text { Guangzhou, } \\
\text { Guangdong, China }\end{array}$ & 200 & $118^{* * *}$ & yes & yes & 10,207 & - tact) & $\begin{array}{c}\text { AUC }=0.740 \\
(0.645,0.836) \\
\text { Accuracy }=0.729\end{array}$ & - \\
\hline Lin et al. [55] & TP-53 & mutation & PWI & $\begin{array}{c}\text { TCGA/TCIA- } \\
\text { BRCA }\end{array}$ & 88 & LOOCV & yes & no & 5234 & - & $\begin{array}{l}\mathrm{AUC}=0.886 \\
(0.817-0.955)\end{array}$ & images \\
\hline
\end{tabular}


Table 3. Cont.

\begin{tabular}{|c|c|c|c|c|c|c|c|c|c|c|c|c|}
\hline Study & Biomarker & Alteration & Modality & Dataset Origin & Training & Validation & $\begin{array}{c}\text { Feature } \\
\text { Reduction }\end{array}$ & $\begin{array}{c}\text { Feature } \\
\text { Robustness }\end{array}$ & $\begin{array}{l}\text { \# Radiomic } \\
\text { Features }\end{array}$ & $\begin{array}{l}\text { Additional } \\
\text { Features }\end{array}$ & $\begin{array}{c}\text { Predictive Power } \\
\text { Measure = Mean (95\% } \\
\text { Confidence Interval) }\end{array}$ & Open Source \\
\hline Ma et al. [56] & Ki-67 & $\begin{array}{l}\text { High } \\
\text { expression at } \\
>14 \%\end{array}$ & PWI & $\begin{array}{l}\text { Tianjin Medical } \\
\text { University Cancer } \\
\text { Institute and } \\
\text { Hospital, National } \\
\text { Clinical Research } \\
\text { Center for Cancer, } \\
\text { Tianjin, China }\end{array}$ & 159 & $10-\mathrm{CV}$ & yes & no & 56 & - & $\begin{array}{c}\text { AUC }=0.773 \\
\text { Accuracy }=0.757\end{array}$ & - \\
\hline Monti et al. [57] & $\begin{array}{l}\text { HER-2, } \\
\text { Ki-67 }\end{array}$ & mutation & PWI & $\begin{array}{l}\text { Hospital of Moscati, } \\
\text { Avellino, Italy; } \\
\text { Institute for } \\
\text { Hospitalization and } \\
\text { Healthcare SDN, } \\
\text { Naples, Italy } \\
\text { Emeroncy }\end{array}$ & $\begin{array}{c}\text { HER-2: } \\
48 \\
\text { Ki-67: } \\
49\end{array}$ & bootstrap & yes & no & 163 & $\begin{array}{l}\text { Pharmacokinetic } \\
\text { maps }\end{array}$ & 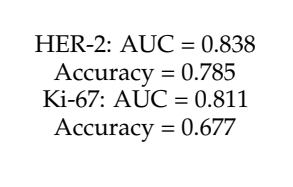 & \\
\hline $\begin{array}{c}\text { Tagliafico et al. } \\
\text { [67] }\end{array}$ & Ki-67 & $\begin{array}{l}\text { High } \\
\text { expression at } \\
>14 \%\end{array}$ & DBT & $\begin{array}{c}\text { Emergency } \\
\text { Radiology, IRCCS } \\
\text { Policlinico San } \\
\text { Martino, Genova, } \\
\text { Italy }\end{array}$ & 70 & bootstrap & yes & no & 106 & - & $\mathrm{AUC}=0.698$ & Code, features \\
\hline Zhang et al. [65] & Ki-67 & $\begin{array}{l}\text { High } \\
\text { expression at } \\
>14 \%\end{array}$ & DWI & $\begin{array}{l}\text { The Second } \\
\text { Hospital, Dalian } \\
\text { Medical University, } \\
\text { Dalian, China }\end{array}$ & 101 & $27^{*}$ & yes & no & 1029 & - & $\begin{array}{c}\mathrm{AUC}=0.72(0.495-0.857) \\
\text { Accuracy }=0.70\end{array}$ & - \\
\hline Zhou et al. [69] & HER-2 & $\begin{array}{c}\text { positive (IHC } \\
\text { 3+) vs. } \\
\text { negative (IHC } \\
0 \text { or 1) } \\
\text { HER-2: }\end{array}$ & DMG & $\begin{array}{l}\text { Henan Provincial } \\
\text { People's Hospital, } \\
\text { Henan, China }\end{array}$ & 244 & $62 *$ & yes & no & 186 & - & $\begin{array}{c}\text { AUC }=0.787 \\
(0.673-0.885) \\
\text { Accuracy }=77.00 \%\end{array}$ & \\
\hline Zhou et al. [62] & $\begin{array}{l}\text { HER-2; } \\
\text { Ki-67 }\end{array}$ & $\begin{array}{c}\text { positive (IHC } \\
3+\text { ) vs. } \\
\text { negative (IHC } \\
0 \text { or 1) } \\
\text { Ki-67: High } \\
\text { expression at } \\
>20 \%\end{array}$ & PWI & $\begin{array}{l}\text { The Affiliated } \\
\text { Huaian No. } 1 \\
\text { People's Hospital } \\
\text { of Nanjing Medical } \\
\text { University, China }\end{array}$ & 126 & $5-\mathrm{CV}$ & yes & yes & 386 & - & $\begin{array}{c}\text { HER-2: AUC }=0.68 \\
\text { Accuracy }=0.60 \\
\text { Ki-67: AUC }=0.74 \\
\text { Accuracy }=0.69\end{array}$ & \\
\hline
\end{tabular}

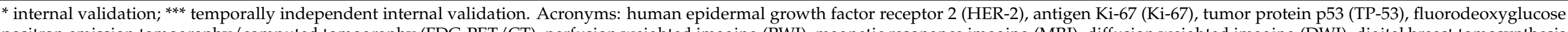

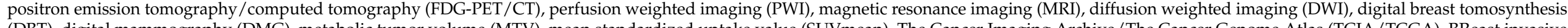

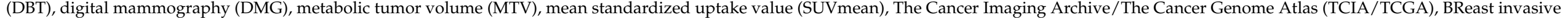

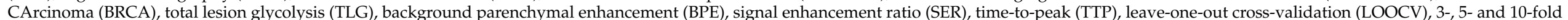
cross-validation (3-, 5- and 10-CV), area under the curve (AUC). 


\subsubsection{HER-2}

Ten studies focused on HER-2 status prediction based on radiomic features derived from PWI $(n=7)$ [54,57-62], DMG $(n=1)$ [69], PET/CT $(n=1)$ [68], and conventional MRI $(n=1)$ [64]. The term HER-2 positivity was used equivalently to an immunohistochemistry (IHC) result of +3 in most studies, while HER-2 negativity corresponded to an IHC score of 0 or +1 .

Among PWI radiomics analysis, the highest predictive performance was achieved by Fan et al. by means of a logistic regression model based on 15 features from dynamic contrast enhanced MRI (DCE-MRI) images ( $\mathrm{AUC}=0.95$, internal validation) [59]. Conventional MR features were also shown to associate with HER-2 status in [64] (accuracy of $73.6 \%$, training cohort). In the study using PET/CT, only mean standardized uptake value (SUV $\mathrm{V}_{\text {mean }}$ ) and total lesion glycolysis (TLG) were independently associated with HER-2 status ( $p=0.021$ and $p=0.046$, respectively) [68]. DMG radiomic features were employed to predict HER-2 status in [69]. Authors reported higher prediction performance when employing a combination of bilateral craniocaudal and mediolateral oblique view images derived from $2 \mathrm{D} \mathrm{MG}$ (AUC $=0.787$, internal validation), compared to radiomic features from each view alone.

\subsubsection{Ki-67}

Three studies investigated the relationship between Ki-67 expression levels and PWI radiomics (AUC $=0.74-0.81$ ) $[56,57,62]$, with two of them employing pharmacokinetic radiomic features. Authors in two of the three studies suggested that high Ki-67 expressing tumors are associated with higher intra-tumoral heterogeneity. On the other hand, DWI radiomics were employed in [65], achieving a final AUC of 0.72 on an internal validation cohort. Another study combined both, PWI and DWI radiomics, to predict Ki-67 status and reported a final AUC of 0.81 after cross-validation by means of a multi-task learning model which was also trained to predict tumor grade [66].

The study using PET/CT could not find any radiomic features that were significantly associated with Ki-67 expression level [68]. Another study used DBT images and showed that a combination of the five most predictive features yielded an AUC of 0.698 for lowversus high Ki-67 expression [67]. Liang et al. compared the utility of T1-weighted with contrast $(\mathrm{T} 1+\mathrm{C})$ to T2-weighted $(\mathrm{T} 2 \mathrm{w})$ radiomics to predict $\mathrm{Ki}-67$ [63]. The analysis revealed that the $\mathrm{T} 2 \mathrm{w}$ image-based radiomics classifier could significantly associate to Ki-67 expression on an external cohort (AUC: 0.740 (95\% CI: 0.645-0.836), whereas T1 + C-based radiomics failed for the same dataset.

Among the eight studies exploring an association of Ki-67 expression levels with breast cancer radiomics, four studies employed a cut-off threshold of 14\% [56,63,65,67], two studies used a threshold of $20 \%[62,68]$ and the other two did not specify any cut-off value.

\subsubsection{TP-53}

The strongest association for breast cancer was found in PWI radiomics of 88 patients in which 13 radiomic features predicted TP-53 alterations with an AUC of 0.886 (95\% CI: 0.817-0.955) after cross-validation [55].

\subsection{Lung Cancer}

\subsubsection{Summary}

In total, 33 studies investigating radiomics and biological endpoints for lung lesions were identified. The imaging modalities employed were CT $(n=22)$ [70-91], PET/CT $(n=8)$ [92-99], PET $(n=2)$ [100,101] and MRI $(n=1)$ [102]. One study investigated radiomics from metastases, while all other studies associated tissue biomarkers with radiomics of the primary tumor. Almost half of the studies selected histological subtypes and used adenocarcinoma patients only $(n=15)$, whereas the other half used a mix of histologies. Research has predominantly focused on EGFR ( $n=26)$ [70-86,92-97,100-102], followed by KRAS $(n=5)$ [83,97,100-102], ALK $(n=3)[87,98,102], \operatorname{PD}-\operatorname{L1}(n=3)[88,89,99]$, 
Ki-67 $(n=2)[90,91]$, and TP-53 $(n=1)$ [84]. All studies showed a significant relationship between EGFR and radiomic features (AUC $=0.66-0.95$ ). Two studies that could not find an association with KRAS status, but all remaining studies found some linkage between radiomics and the respective biomarker (AUC $=0.66-0.99$ ). In total, 21 studies validated their predictive models, two of which were external validation. None of the studies reported any radiomics quality measure, and only one of them was a registered prospective study [83]. A summary of the findings of this section can be found in Table 4 . 
Table 4. An overview of the radiomic studies included in the lung cancer section.

\begin{tabular}{|c|c|c|c|c|c|c|c|c|c|c|c|c|}
\hline Study & Biomarker & Alteration & Modality & Dataset Origin & Training & Validation & $\begin{array}{c}\text { Feature } \\
\text { Reduction }\end{array}$ & $\begin{array}{c}\text { Feature } \\
\text { Robustness }\end{array}$ & $\begin{array}{l}\text { \# Radiomic } \\
\text { Features }\end{array}$ & $\begin{array}{l}\text { Additional } \\
\text { Features }\end{array}$ & $\begin{array}{c}\text { Predictive Power } \\
\text { Measure = Mean }(95 \% \\
\text { Confidence Interval) }\end{array}$ & Open Source \\
\hline Aerts et al. [70] & EGFR & $\begin{array}{l}\text { Exons } 19 \text { and } \\
21 \text { mutations }\end{array}$ & CT & $\begin{array}{c}\text { Memorial } \\
\text { Sloan-Kettering } \\
\text { Cancer Center, } \\
\text { New York City, } \\
\text { New York, US }\end{array}$ & 47 & - & yes & yes & 183 & - & AUC $=0.91$ & Images \\
\hline Chen et al. [102] & $\begin{array}{c}\text { EGFR; } \\
\text { KRAS; ALK }\end{array}$ & mutation & MR & $\begin{array}{l}\text { City of Hope } \\
\text { Medical Center, } \\
\text { Duarte, } \\
\text { California, US }\end{array}$ & 110 & LOOCV & yes & yes & 2786 & $\begin{array}{c}\text { Age; sex; ethnicity; } \\
\text { history of smoking; } \\
\text { histology type; } \\
\text { other metastatic } \\
\text { sites }\end{array}$ & $\begin{array}{c}\text { EGFR: AUC }=0.912 \\
\text { Accuracy }=77.7 \% \\
\text { ALK: AUC }=0.915 \\
\text { Accuracy }=86.7 \% \\
\text { KRAS: AUC }=0.985 \\
\text { Accuracy }=96.7 \%\end{array}$ & - \\
\hline Gu et al. [91] & Ki-67 & $\begin{array}{l}\text { High Ki-67 } \\
\text { expression as } \\
>50 \%\end{array}$ & CT & $\begin{array}{l}\text { The Third } \\
\text { Xiangya Hospital } \\
\text { of Central South } \\
\text { University, } \\
\text { Hunan, China }\end{array}$ & 245 & $10-\mathrm{CV}$ & yes & no & 103 & $\begin{array}{c}\text { Lobulation sign; } \\
\text { spicule sign; } \\
\text { cavitation; cystic } \\
\text { necrosis; pleural } \\
\text { indentation; pleural } \\
\text { effusion }\end{array}$ & $\mathrm{AUC}=0.782$ & - \\
\hline Hong et al. [85] & EGFR & $\begin{array}{l}\text { Exons } 18,19 \\
20, \text { and } 21 \\
\text { mutations }\end{array}$ & CT & $\begin{array}{l}\text { The First } \\
\text { Hospital of } \\
\text { China Medical } \\
\text { University, } \\
\text { Shenyang, China }\end{array}$ & 140 & $61^{*}$ & yes & no & 396 & $\begin{array}{l}\text { Age; sex; history of } \\
\text { smoking }\end{array}$ & $\begin{array}{c}\text { AUC }=0.851 \\
(0.750-0.951) \\
\text { c-index }=0.835 \\
(0.825-0.845)\end{array}$ & - \\
\hline Huang et al. [71] & EGFR & mutation & CT & $\begin{array}{l}\text { The University of } \\
\text { Texas MD } \\
\text { Anderson Cancer } \\
\text { Center, Houston, } \\
\text { Texas }\end{array}$ & 46 & - & yes & yes & 89 & - & $\mathrm{AUC}=0.88$ & Images \\
\hline Jia et al. [72] & EGFR & $\begin{array}{l}\text { Exons } 19 \text { and } \\
21 \text { mutations }\end{array}$ & CT & $\begin{array}{l}\text { Shanghai Chest } \\
\text { Hospital, } \\
\text { Shanghai, China } \\
\text { Shanghai }\end{array}$ & 345 & $158^{*}$ & no & no & 440 & $\begin{array}{l}\text { Age; sex; smoking } \\
\text { history; TNM stage }\end{array}$ & $\begin{array}{l}\text { AUC }=0.828 \\
(0.764-0.892)\end{array}$ & - \\
\hline Jiang et al. [99] & PD-L1 & $\begin{array}{l}\text { PD-L1 cutoff } \\
\text { value of 1\% } \\
\text { and } 50 \%\end{array}$ & $\mathrm{PET} / \mathrm{CT}$ & $\begin{array}{l}\text { Institute of } \\
\text { Medical Imaging, } \\
\text { Zhongshan } \\
\text { Hospital of } \\
\text { Fudan University, } \\
\text { Shanghai, China }\end{array}$ & 266 & $133 *$ & yes & no & 1744 & $\begin{array}{l}\text { SUVmax; age; sex; } \\
\text { smoking status; } \\
\text { TNM stage; } \\
\text { histology type }\end{array}$ & $\mathrm{AUC}=0.97$ & - \\
\hline Jiang et al. [92] & EGFR & mutation & $\mathrm{PET} / \mathrm{CT}$ & $\begin{array}{l}\text { Shanghai } \\
\text { Institute of } \\
\text { Medical Imaging, } \\
\text { Zhongshan } \\
\text { Hospital of } \\
\text { Fudan University, } \\
\text { Shanghai, China }\end{array}$ & 80 & $10-\mathrm{CV}$ & yes & no & 512 & $\begin{array}{l}12 \text { semantic } \\
\text { features }\end{array}$ & $\mathrm{AUC}=0.953$ & - \\
\hline Koyasu et al. [93] & EGFR & mutation & $\mathrm{PET} / \mathrm{CT}$ & $\begin{array}{l}\text { TCIA- NSCLC } \\
\text { Radiogenomics }\end{array}$ & 138 & $10-\mathrm{CV}$ & yes & no & Not disclosed & $\begin{array}{l}\text { SUVmax; } \\
\text { SUVmean; TLG; } \\
\text { MTV }\end{array}$ & $\begin{array}{c}\mathrm{AUC}=0.659 \\
\text { Accuracy }=81.2 \%\end{array}$ & Images, ROI \\
\hline
\end{tabular}


Table 4. Cont.

\begin{tabular}{|c|c|c|c|c|c|c|c|c|c|c|c|c|}
\hline Study & Biomarker & Alteration & Modality & Dataset Origin & Training & Validation & $\begin{array}{c}\text { Feature } \\
\text { Reduction }\end{array}$ & $\begin{array}{c}\text { Feature } \\
\text { Robustness }\end{array}$ & $\begin{array}{l}\text { \# Radiomic } \\
\text { Features }\end{array}$ & $\begin{array}{l}\text { Additional } \\
\text { Features }\end{array}$ & $\begin{array}{c}\text { Predictive Power } \\
\text { Measure = Mean (95\% } \\
\text { Confidence Interval) }\end{array}$ & Open Source \\
\hline Li et al. [94] & EGFR & $\begin{array}{c}\text { Exons 18-24 } \\
\text { mutations }\end{array}$ & PET/CT & $\begin{array}{l}\text { Tianjin Medical } \\
\text { University } \\
\text { Cancer Hospital, } \\
\text { Tianjin, China }\end{array}$ & 115 & $10-\mathrm{CV}$ & yes & no & 38 & $\begin{array}{c}\text { SUVmax; } \\
\text { SUVmean; } \\
\text { SUVpeak; TLG; } \\
\text { MTV; age; sex; } \\
\text { smoking status; } \\
\text { TNM stage; lesion } \\
\text { location }\end{array}$ & $\begin{array}{c}\text { AUC }=0.822 \\
\text { Accuracy }=82.65 \%\end{array}$ & - \\
\hline Li et al. [74] & EGFR & mutation & CT & $\begin{array}{l}\text { Second Xiangya } \\
\text { Hospital of } \\
\text { Central South } \\
\text { University, } \\
\text { Hunan, China }\end{array}$ & 51 & $10-\mathrm{CV}$ & yes & yes & 1695 & - & $\mathrm{AUC}=0.83(0.68-0.92)$ & Images, ROI \\
\hline Li et al. [75] & EGFR & $\begin{array}{l}\text { Exon } 19 \text { and } 21 \\
\text { mutations }\end{array}$ & СТ & $\begin{array}{l}\text { Shanghai Chest } \\
\text { Hospital, } \\
\text { Shanghai, China }\end{array}$ & 810 & $200 *$ & yes & no & 440 & $\begin{array}{l}\text { DL prediction; age; } \\
\text { sex; smoking } \\
\text { history; } \\
\text { pathological stage }\end{array}$ & $\begin{array}{l}\text { AUC }=0.834 \\
(0.776-0.892)\end{array}$ & - \\
\hline Li et al. [76] & EGFR & $\begin{array}{l}\text { Exon } 19 \text { and } 21 \\
\text { mutations }\end{array}$ & CT & $\begin{array}{l}\text { Shengiing } \\
\text { Hospital of } \\
\text { China Medical } \\
\text { University, } \\
\text { Liaoning, China }\end{array}$ & 236 & $76^{* * *}$ & yes & yes & 580 & $\begin{array}{l}\text { Age; sex; tumor } \\
\text { grade; lobe; } \\
\text { smoking history; } \\
\text { intrapulmonary } \\
\text { metastasis } \\
10 \text { tumor spatial }\end{array}$ & $\mathrm{AUC}=0.7750-0.7925$ & - \\
\hline Liu et al. [86] & EGFR & $\begin{array}{l}\text { Exons } 18-21 \\
\text { mutations }\end{array}$ & CT & $\begin{array}{l}\text { Tianjin Medical } \\
\text { University } \\
\text { Cancer Institute } \\
\text { and Hospital, } \\
\text { Tianjin, China }\end{array}$ & 298 & bootstrap & yes & yes & 1025 & $\begin{array}{l}\text { location features; } \\
\text { age; sex; } \\
\text { histological } \\
\text { subtype; } \\
\text { pathological stage; } \\
\text { smoking history } \\
45 \text { categorical } \\
\text { variables including: } \\
\text { age, sex, smoking } \\
\text { status, CEA level, } \\
\text { vascular infiltration, } \\
\text { visceral pleural } \\
\text { infiltration, lymph } \\
\text { node metastasis, } \\
\text { histological } \\
\text { subtype, } \\
\text { pathological stage, } \\
\text { type of lesion, } \\
\text { tumor location, } \\
\text { tumor size, tumor } \\
\text { necrosis, lobulation, } \\
\text { spiculation, } \\
\text { vacuolization, etc. }\end{array}$ & $\begin{array}{l}\mathrm{AUC}=0.709 \\
(0.654-0.766)\end{array}$ & code \\
\hline
\end{tabular}


Table 4. Cont.

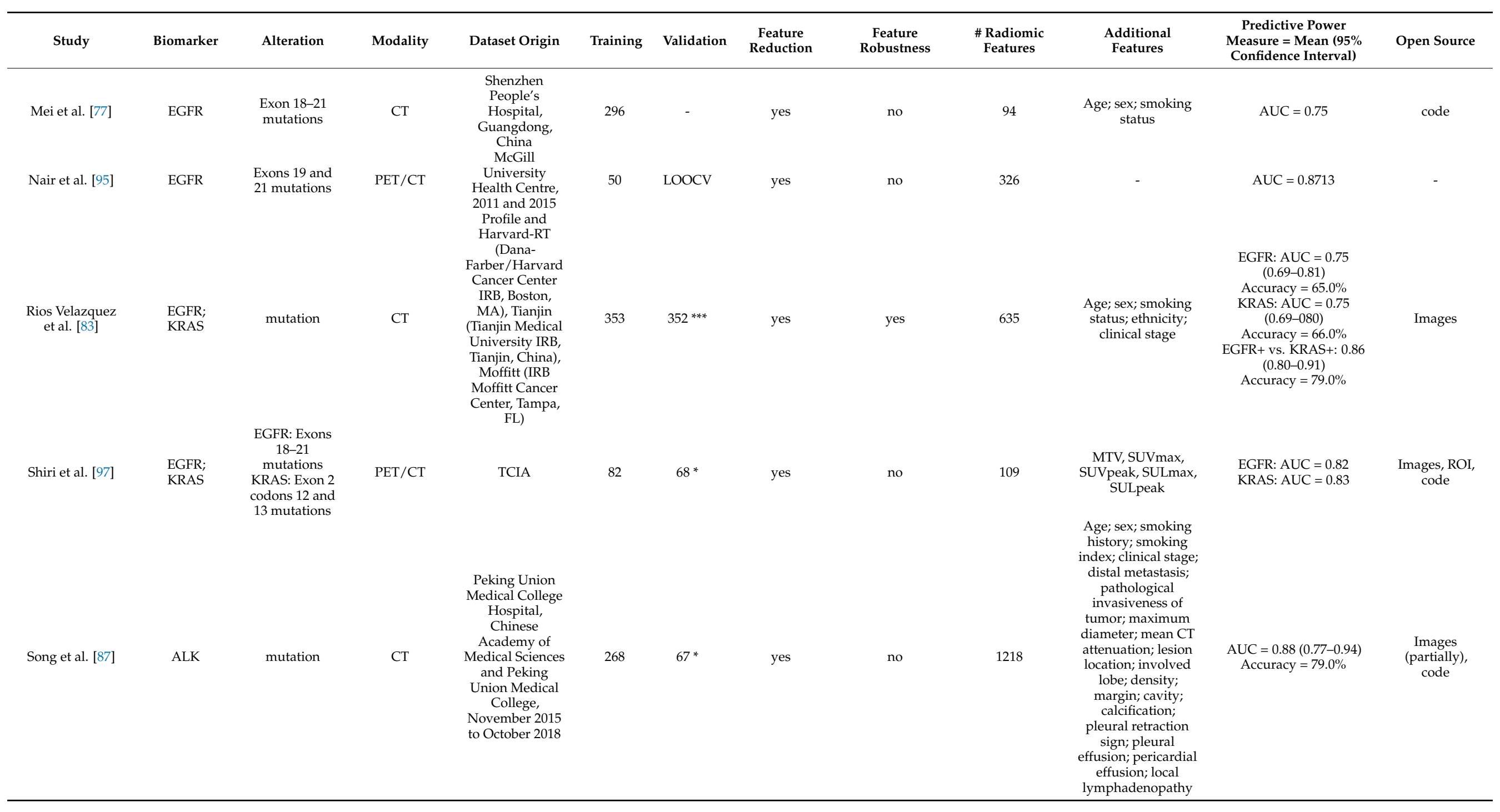


Table 4. Cont.

\begin{tabular}{|c|c|c|c|c|c|c|c|c|c|c|c|c|}
\hline Study & Biomarker & Alteration & Modality & Dataset Origin & Training & Validation & $\begin{array}{c}\text { Feature } \\
\text { Reduction }\end{array}$ & $\begin{array}{c}\text { Feature } \\
\text { Robustness }\end{array}$ & $\begin{array}{l}\text { \# Radiomic } \\
\text { Features }\end{array}$ & $\begin{array}{l}\text { Additional } \\
\text { Features }\end{array}$ & $\begin{array}{c}\text { Predictive Power } \\
\text { Measure = Mean }(95 \% \\
\text { Confidence Interval) }\end{array}$ & Open Source \\
\hline Sun et al. [88] & PD-L1 & $\begin{array}{l}\text { High PD-L1 } \\
\text { expression as } \\
\geq 50 \%\end{array}$ & CT & $\begin{array}{c}\text { The First } \\
\text { Affiliated } \\
\text { Hospital of } \\
\text { Soochow } \\
\text { University, } \\
\text { Suzhou City, } \\
\text { China }\end{array}$ & 260 & $130 *$ & yes & yes & 200 & $\begin{array}{c}\text { Age; sex; tumor } \\
\text { location; CEA level; } \\
\text { TNM stage; } \\
\text { smoking status; } \\
\text { histologic type; } \\
\text { histologic grade }\end{array}$ & $\mathrm{AUC}=0.848$ & - \\
\hline Tu et al. [78] & EGFR & $\begin{array}{l}\text { Exons } 18-21 \\
\text { mutations }\end{array}$ & CT & $\begin{array}{l}\text { Changzheng } \\
\text { Hospital, Second } \\
\text { Military Medical } \\
\text { University, } \\
\text { Shanghai, China }\end{array}$ & 243 & $130 *$ & yes & yes & 234 & $\begin{array}{c}\text { Age; sex; smoking } \\
\text { status; CEA level; } \\
\text { clinical stage; } \\
\text { maximum } \\
\text { diameter; density; } \\
\text { tumor location; } \\
\text { interface; shape; } \\
\text { lobulation; pleural } \\
\text { indentation; } \\
\text { spiculation; cusp } \\
\text { angle; spine-like } \\
\text { process; vacuole } \\
\text { sign; cavity sign; air } \\
\text { bronchograms; } \\
\text { vascular } \\
\text { convergence; } \\
\text { pleura thickening; } \\
\text { pleural effusion; } \\
\text { lymphadenopathy } \\
\text { 78 clinical and }\end{array}$ & $\begin{array}{c}\text { AUC }=0.818 \\
(0.751-0885) \\
\text { Accuracy }=75.8 \%\end{array}$ & - \\
\hline Wang et al. [84] & EGFR; TP-53 & mutation & CT & $\begin{array}{l}\text { Nanjing Medical } \\
\text { University } \\
\text { Affiliated Cancer } \\
\text { Hospital, } \\
\text { Nanjing, China }\end{array}$ & 41 & $20^{*}$ & yes & no & 718 & $\begin{array}{l}78 \text { clinical and } \\
\text { pathological } \\
\text { features (age, sex, } \\
\text { smoking status, } \\
\text { histological } \\
\text { subtypes, } \\
\text { pathological stages, } \\
\text { etc.) }\end{array}$ & $\begin{array}{l}\text { EGFR: AUC }=0.697 \\
\text { TP-53: AUC }=0.656\end{array}$ & code \\
\hline Yang et al. [79] & EGFR & $\begin{array}{l}\text { Exons 18-21 } \\
\text { mutations }\end{array}$ & CT & $\begin{array}{l}\text { The First } \\
\text { Affiliated } \\
\text { Hospital of } \\
\text { Guangzhou } \\
\text { Medical } \\
\text { University, } \\
\text { Guangzhou, } \\
\text { China }\end{array}$ & 306 & $161^{* * *}$ & yes & no & 1063 & $\begin{array}{l}\text { Age; sex; smoking } \\
\text { history; CT pattern; } \\
\text { histopathological } \\
\text { subtype }\end{array}$ & $\begin{array}{l}\text { AUC }=0.779 \\
(0.702-0.856)\end{array}$ & code \\
\hline
\end{tabular}


Table 4. Cont.

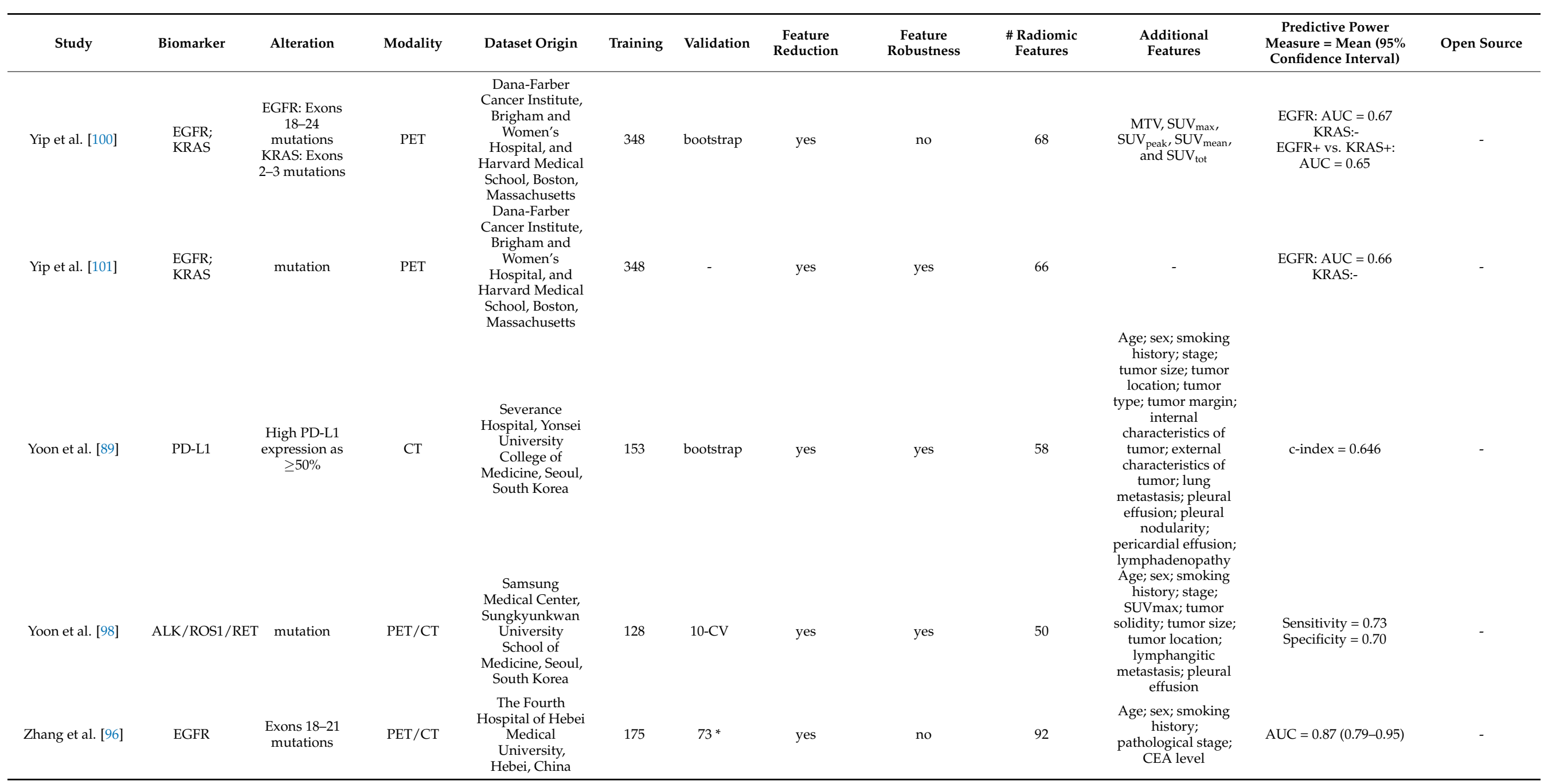


Table 4. Cont.

\begin{tabular}{|c|c|c|c|c|c|c|c|c|c|c|c|c|}
\hline Study & Biomarker & Alteration & Modality & Dataset Origin & Training & Validation & $\begin{array}{c}\text { Feature } \\
\text { Reduction }\end{array}$ & $\begin{array}{c}\text { Feature } \\
\text { Robustness }\end{array}$ & $\begin{array}{l}\text { \# Radiomic } \\
\text { Features }\end{array}$ & $\begin{array}{l}\text { Additional } \\
\text { Features }\end{array}$ & $\begin{array}{c}\text { Predictive Power } \\
\text { Measure = Mean (95\% } \\
\text { Confidence Interval) }\end{array}$ & Open Source \\
\hline Zhang et al. [80] & EGFR & $\begin{array}{l}\text { Exons 18-21 } \\
\text { mutations }\end{array}$ & CT & $\begin{array}{l}\text { West China } \\
\text { Hospital, } \\
\text { Sichuan, China }\end{array}$ & 140 & $40^{*}$ & yes & no & 485 & $\begin{array}{l}\text { Age; sex; smoking } \\
\text { status }\end{array}$ & $\begin{array}{c}\text { AUC }=0.8725 \\
\text { Accuracy }=72.5 \%\end{array}$ & - \\
\hline Zhao et al. [82] & EGFR & $\begin{array}{l}\text { Exons 18-21 } \\
\text { mutations }\end{array}$ & CT & $\begin{array}{c}\text { Huadong } \\
\text { Hospital } \\
\text { Affiliated to } \\
\text { Fudan University, } \\
\text { Shanghai, China; } \\
\text { TCIA }\end{array}$ & $\begin{array}{c}464 \\
\text { nodules }\end{array}$ & $\begin{array}{c}115 \\
\text { nodules } \\
* \\
37 \\
\text { nodules } \\
* *\end{array}$ & yes & yes & 475 & DL prediction & $\mathrm{AUC}=0.76$ & Images, ROI \\
\hline Zhou et al. [90] & Ki-67 & $\begin{array}{c}\text { High Ki-67 } \\
\text { expression as > } \\
40 \%\end{array}$ & CT & $\begin{array}{l}\text { Tianjin Medical } \\
\text { University } \\
\text { Cancer Institute } \\
\text { and Hospital, } \\
\text { Tianjin, China }\end{array}$ & 110 & - & yes & no & 105 & $\begin{array}{l}\text { Age; sex; smoking } \\
\text { history; histological } \\
\text { subtype; TNM } \\
\text { stage }\end{array}$ & $\mathrm{AUC}=0.77$ & code \\
\hline
\end{tabular}

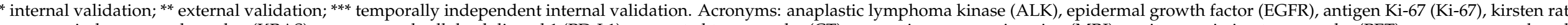

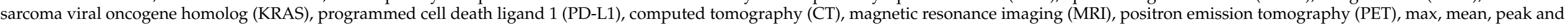

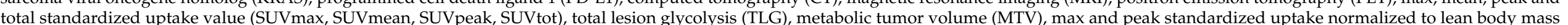

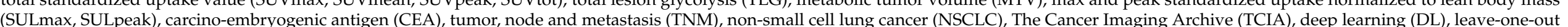
cross-validation (LOOCV), 3-, 5- and 10-fold cross-validation (3-, 5- and 10-CV), area under the curve (AUC). 


\subsubsection{EGFR}

The power of CT radiomics to predict EGFR mutations status strongly varied among the studies using validation (AUC $=0.69-0.851$ ) $[72-76,78-86]$. Across CT radiomics studies, relevant features for EGFR mutation status prediction were associated with texture heterogeneity, suggesting that mutated tumors were more heterogeneous. Further, two studies observed an association of $\mathrm{CT}$ radiomics with EGFR mutation subtypes, i.e., differentiation subtype to wildtype (AUC $=0.655-0.727$ ) [76,77] and within subtypes (AUC $=0.708-0.87)$ [81,95]. While PET radiomics also showed potential for EGFR mutation status prediction (AUC $=0.67$, internal validation) $[100,101]$, combining $C T$ and PET were reported similar or better compared to single modal radiomics models [92-96]. Two studies examined radiomics at different time points other than pre-treatment [70,71]. In contrast to three-week post-treatment CT radiomics, one delta radiomic feature (i.e., change of feature value over time) was found predictive for EGFR mutation status (AUC $=0.74$, weakly corrected to delta volume and diameter) [70]. Multiple studies were found using contrast-enhanced CT (CE CT) $[74,84,85]$, non-CE CT imaging [70,72,73,77-79,81,82] or a mix of thereof $[75,83]$. In a recent study, it was shown that a model based on CE CT features did not significantly performed different to a model based non-CE CT for EGFR mutation status prediction [85].

\subsubsection{KRAS}

CT radiomics was weakly predictive for KRAS mutation status in 763 lung adenocarcinoma patients from four institutions ( $\mathrm{AUC}=0.63$, temporally independent validation) [83] Authors suggested that KRAS mutant tumors were more homogeneous. PET radiomics was reported non-predictive for KRAS mutation status (AUC $<0.56$, no validation) $[100,101]$. Radiomics was further shown to better differentiate between EGFR and KRAS mutated tumors in CT (AUC $=0.80$, internal validation) [83] than in PET (AUC $=0.65)$ [100].

\subsubsection{ALK}

For ALK mutation status, CT radiomics showed an AUC of 0.80 on a temporally independent validation cohort [87]. Selected radiomic features inferred that ALK mutated tumors were associated with denser tumors. One study observed that PET-based radiomics combined with tumor stage and age was able to differentiate ALK/ROS1/RET fusionpositive and fusion negative tumors (sensitivity $=0.73$, specificity $=0.70$ ) [98]. A study on 110 patients evaluated if MR radiomics from brain metastasis originated from lung cancer was shown to associate with EGFR, ALK and KRAS mutations and reported excellent model performances for all three tissue biomarkers (AUC > 0.9, LOOCV) [102].

\subsubsection{PD-L1}

PD-L1 expression levels were observed to associate with CT radiomic features in two studies (AUC $=0.661$ [89] and AUC $=0.848$ [88], internal validations), indicating that dense and homogeneous tumors (without ground-glass opacity, necrosis, cavitation or calcification) were more likely PD-L1 positive in lung adenocarcinoma [89]. Radiomics from $\mathrm{PET} / \mathrm{CT}$ imaging was found to be similarly strongly predictive as CT but outperformed PET in PD-L1 expression level prediction for 399 stage I-IV non-small cell lung cancer (NSCLC) patients (AUC > 0.8, internal validation) [99].

\subsubsection{Ki-67}

$\mathrm{CT}$ radiomic features were found significantly predictive for Ki-67 in two studies (best performing feature: inverse variance, $\mathrm{AUC}=0.77)[90,91]$.

\subsubsection{TP-53}

The association between CT radiomic features and TP-53 mutation was studied in [84]. Authors reported a final AUC of 0.604 on an internal validation cohort. 


\subsection{Gastrointestinal Cancer}

\subsubsection{Summary}

A total of ten studies addressing the relationship of radiomics and biological tissue markers in gastrointestinal cancers were identified. The imaging modalities used were $\mathrm{CT}$ $(n=6)$ [103-107], combined PET/CT $(n=2)[108,109]$, MRI $(n=2)[110,111]$ and MRI + PWI + DWI $(n=1)$ [112]. The tumor types analyzed belong to gastric cancers $(n=2)[103,104]$, rectal cancers $(n=3)$ [110-112], pancreatic cancers $(n=2)[105,108]$, colorectal cancer $(C R C)$ and colorectal liver metastases $(n=3)[106,107,109]$. The most frequent biomarker analyzed was KRAS mutation $(n=7)$ [106-112] followed by TP-53 mutation $(n=2)[108,109]$, Ki-67 $(n=3)[104,105,112]$ and HER-2 expression status $(n=2)[103,112]$. One group studied BRAF $(n=1)$ together with KRAS and NRAS as one mutation signature [106]. All except one study on TP- 53 showed a significant correlation between the respective biomarker and radiomic features (AUC $=0.65-0.88$ ). All studies were set up retrospectively and used internal data. Two research groups validated their results on external datasets. None of the studies reported any radiomics quality measure and none of them were registered prospective studies. A summary of the findings of this section can be found in Table 5 . 
Table 5. An overview of the radiomic studies included in the gastrointestinal cancer section.

\begin{tabular}{|c|c|c|c|c|c|c|c|c|c|c|c|c|}
\hline Study & Biomarker & Alteration & Modality & Dataset Origin & Training & Validation & $\begin{array}{c}\text { Feature } \\
\text { Reduction }\end{array}$ & $\begin{array}{c}\text { Feature } \\
\text { Robustness }\end{array}$ & $\begin{array}{l}\text { \# Radiomic } \\
\text { Features }\end{array}$ & $\begin{array}{l}\text { Additional } \\
\text { Features }\end{array}$ & $\begin{array}{c}\text { Predictive Power } \\
\text { Measure = Mean (95\% } \\
\text { Confidence Interval) }\end{array}$ & Open Source \\
\hline Chen et al. [109] & KRAS; TP-53 & $\begin{array}{l}\text { KRAS: Exon } 2 \\
\text { codons } 12 \text { and } \\
13 \text { mutation } \\
\text { TP-53: Exons } \\
2-11 \text { mutations }\end{array}$ & $\begin{array}{l}\text { FDG- } \\
\text { PET/CT }\end{array}$ & $\begin{array}{c}\text { China Medical } \\
\text { University } \\
\text { Hospital, } \\
\text { Taichung, Taiwan }\end{array}$ & 74 & - & yes & no & 56 & $\begin{array}{l}\text { SUVmax, SUVpeak, } \\
\text { SUVtot, MTV, } \\
\text { TLGmax, TLGpeak, } \\
\text { and TLGmean }\end{array}$ & $\begin{array}{c}\text { KRAS: AUC }=0.79 \\
\text { Accuracy }=77 \% \\
\text { TP-53: AUC }=0.71 \\
\text { Accuracy }=62 \%\end{array}$ & - \\
\hline Cui et al. [110] & KRAS & $\begin{array}{l}\text { KRAS: Exons } \\
\text { 2-4 mutations }\end{array}$ & MRI & $\begin{array}{l}\text { Shanxi Province } \\
\text { Cancer Hospital, } \\
\text { Taiyuan, China; } \\
\text { Xinhua Hospital, } \\
\text { Shanghai, China }\end{array}$ & 213 & $\begin{array}{l}91^{*} \\
86^{* *}\end{array}$ & yes & no & 960 & - & $\begin{array}{c}\mathrm{AUC}^{*}=0.682 \\
(0.569-0.794) \\
\text { AUC } C^{* *}=0.714 \\
(0.602-0.827)\end{array}$ & - \\
\hline Li et al. [103] & HER-2 & $\begin{array}{c}\text { positive } \\
\text { (HER-2/CE17 } \\
\geq 2 \text { ) vs. } \\
\text { negative } \\
\text { (HER-2/CEP17 } \\
<2 \text { ) }\end{array}$ & CT & $\begin{array}{l}\text { Guangdong } \\
\text { Provincial } \\
\text { People's } \\
\text { Hospital, } \\
\text { Guangzhou, } \\
\text { China }\end{array}$ & 94 & $40^{*}$ & yes & yes & 12,410 & CEA level & $\begin{array}{c}\text { AUC }=0.771 \\
(0.607-0.934)\end{array}$ & - \\
\hline Liang et al. [105] & Ki-67 & mutation & $\mathrm{CT}$ & $\begin{array}{c}\text { The First } \\
\text { Affiliated } \\
\text { Hospital, } \\
\text { Hangzhou, } \\
\text { Zhejiang, China; } \\
\text { Second Affiliated } \\
\text { Hospital, } \\
\text { Hangzhou, } \\
\text { Zhejiang, China }\end{array}$ & 86 & $51^{* *}$ & yes & no & 467 & Clinical stage & $\begin{array}{l}\text { Significant correlation } \\
\quad(p<0.0001)\end{array}$ & - \\
\hline Lim et al. [108] & KRAS; TP-53 & mutation & $\begin{array}{l}\text { FDG- } \\
\text { PET/CT }\end{array}$ & $\begin{array}{c}\text { Samsung } \\
\text { Medical Center, } \\
\text { Sungkyunkwan } \\
\text { University } \\
\text { School of } \\
\text { Medicine, } \\
\text { Gangnam-gu, } \\
\text { Seoul, South } \\
\text { Korea }\end{array}$ & 48 & - & no & yes & 27 & $\begin{array}{l}\text { SUVmax, } \\
\text { SUVmean, SUVstd, } \\
\text { SUVkurt, SUVskew, } \\
\text { SUVent, MTV, TLG }\end{array}$ & $\begin{array}{c}\text { KRAS: AUC }=0.829 \\
\text { TP-53:- }\end{array}$ & $\begin{array}{c}\text { Code } \\
\text { (partially) }\end{array}$ \\
\hline Meng et al. [112] & $\begin{array}{l}\text { Ki-67, KRAS, } \\
\text { HER-2 }\end{array}$ & $\begin{array}{l}\text { KRAS: exon } 2 \\
\text { codons } 12 \text { and } \\
13 \text { mutation } \\
\text { Ki-67: High } \\
\text { expression at } \\
>40 \% \\
\text { HER-2: }\end{array}$ & $\begin{array}{l}\text { MRI, DWI, } \\
\text { PWI }\end{array}$ & $\begin{array}{l}\text { Sixth Affiliated } \\
\text { Hospital of Sun } \\
\text { Yat-sen } \\
\text { University. } \\
\text { Guangzhou, } \\
\text { China }\end{array}$ & 197 & $148^{* * *}$ & yes & yes & 2534 & - & $\begin{array}{c}\text { HER-2: } \text { AUC }=0.696 \\
(0.610-0.782) \\
\text { Accuracy }=0.621 \\
\text { Ki-67: AUC }=0.699 \\
(0.611-0.788) \\
\text { Accuracy }=0.582 \\
\text { KRAS: AUC }=0.651 \\
(0.539-0.763) \\
\text { Accuracy }=0.616\end{array}$ & - \\
\hline
\end{tabular}


Table 5. Cont.

\begin{tabular}{|c|c|c|c|c|c|c|c|c|c|c|c|c|}
\hline Study & Biomarker & Alteration & Modality & Dataset Origin & Training & Validation & $\begin{array}{c}\text { Feature } \\
\text { Reduction }\end{array}$ & $\begin{array}{c}\begin{array}{c}\text { Feature } \\
\text { Robustness }\end{array}\end{array}$ & $\begin{array}{c}\text { \# Radiomic } \\
\text { Features }\end{array}$ & $\begin{array}{l}\text { Additional } \\
\text { Features }\end{array}$ & $\begin{array}{c}\text { Predictive Power } \\
\text { Measure = Mean (95\% } \\
\text { Confidence Interval) }\end{array}$ & Open Source \\
\hline Oh et al. [111] & KRAS & $\begin{array}{l}\text { A59T, G12A, } \\
\text { G12C, G12D, } \\
\text { G12F, G12R, } \\
\text { G12S, G12V, } \\
\text { G13D, G61H, } \\
\text { and Q61 } \\
\text { mutation }\end{array}$ & MRI & $\begin{array}{c}\text { Research } \\
\text { Institute and } \\
\text { Hospital, } \\
\text { National Cancer } \\
\text { Center, Goyang, } \\
\text { Korea }\end{array}$ & 60 & - & no & no & 44 & - & $\begin{array}{c}\text { AUC }=0.884 \\
\text { Accuracy }=81.7 \%\end{array}$ & - \\
\hline Wu et al. [107] & KRAS & $\begin{array}{l}\text { Exons 2-4 } \\
\text { mutations }\end{array}$ & CT & $\begin{array}{l}\text { South China } \\
\text { University of } \\
\text { Technology, } \\
\text { Guangzhou, } \\
\text { Guangdong } \\
\text { Province, China } \\
\text { National Cancer }\end{array}$ & 279 & $119^{* * *}$ & yes & yes & 2634 & 2208 DL features & $\begin{array}{c}\text { c-index }=0.832 \\
(0.762-0.905)\end{array}$ & - \\
\hline Yang et al. [106] & $\begin{array}{l}\text { KRAS; } \\
\text { BRAF }\end{array}$ & $\begin{array}{l}\text { KRAS: Exons } \\
\text { 2-4 mutations } \\
\text { BRAF: v600E } \\
\text { mutation }\end{array}$ & CT & $\begin{array}{c}\text { Center/Cancer } \\
\text { Hospital, } \\
\text { Chinese } \\
\text { Academy of } \\
\text { Medical Sciences } \\
\text { and Peking } \\
\text { Union Medical } \\
\text { College, Beijing, }\end{array}$ & 61 & $57^{* * *}$ & yes & yes & 346 & - & $\begin{array}{c}\text { AUC }=0.829 \\
(0.718-0.939) \\
\text { Accuracy }=0.750 \\
(0.623-0.845)\end{array}$ & - \\
\hline Zhang et al. [104] & Ki-67 & $\begin{array}{c}\text { High } \\
\text { expression as } \\
\geq 10 \%\end{array}$ & CT & $\begin{array}{c}\text { China } \\
\text { Renji Hospital, } \\
\text { Huangpu, } \\
\text { Shanghai, China; } \\
\text { Zhongshan } \\
\text { Hospital, } \\
\text { Shanghai, China; } \\
\text { Sir Run Shaw } \\
\text { Hospital, } \\
\text { Hangzhou, } \\
\text { Zhejiang, China } \\
\text { and First } \\
\text { Affiliated } \\
\text { Hospital of } \\
\text { Wenzhou } \\
\text { Medical } \\
\text { University, } \\
\text { Wenzhou, China }\end{array}$ & 148 & $\begin{array}{c}41^{*} \\
150^{* *}\end{array}$ & yes & yes & 833 & Tumor size & $\begin{array}{c}\text { AUC } *=0.828 \\
(0.681-0.974) \\
\text { AUC } C^{* *}=0.784 \\
(0.701-0.868) \\
\text { Accuracy }{ }^{*}=68.29 \% \\
\text { Accuracy }^{* *}=73.33 \%\end{array}$ & $\begin{array}{l}\text { Images/data } \\
\text { on request }\end{array}$ \\
\hline
\end{tabular}

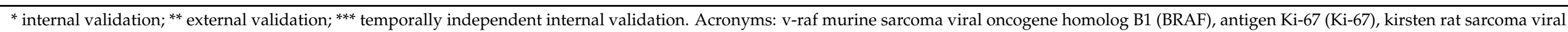

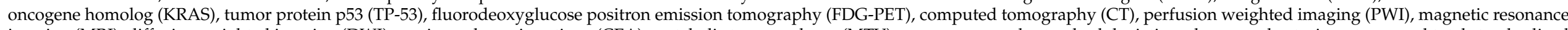

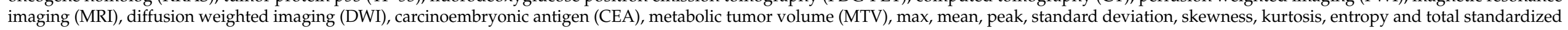

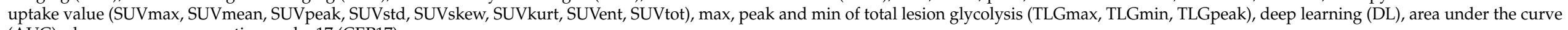
(AUC), chromosome enumeration probe 17 (CEP17) 


\subsubsection{KRAS}

The association of KRAS mutations with radiomic signatures was the most frequently assessed in gastrointestinal cancer. The strongest relationship was found in CE CT of CRC patients, where the mutation signature KRAS/BRAF/NRAS was significantly associated with three GLCM features (energy, maximum probability and sum average), achieving a final AUC of 0.829 on an internal validation cohort [106].

One group focused on the association of KRAS mutation to FDG-PET radiomics of pancreatic ductal adenocarcinoma patients [108], concluding that low-intensity textural features were significantly associated with KRAS gene mutational status (AUC $=0.794-0.82$, training). Authors suggested that KRAS-mutated genes were associated with higher intratumoral heterogeneity levels. The relationship between FDG-PET radiomics and KRAS mutation was also studied for CRC patients in [109]. KRAS-mutated tumors presented an increased value at the 25th percentile of maximal SUV ( $\left.\mathrm{SUV}_{\max }\right)$ of the metabolic tumor volume (MTV) as well as for the GLCM-derived contrast (AUC $=0.73-0.79$, training).

Another study evaluated the association between KRAS mutation and CT imaging features, including hand-crafted and deep learning radiomics, of CRC patients [107]. The combined model achieved the highest performance (c-index $=0.831$ (95\% CI, 0.762-0.905), external validation), when compared to radiomics-alone and deep learning radiomicsalone models.

Two studies evaluated the association between T2w MR radiomics and KRAS mutational status in rectal cancer. In the first one, authors reported a final AUC of 0.884 on the training cohort by means of a decision tree based on three textural features [111]. In the second study, seven features were shown to associate to KRAS mutation status [110]. The best prediction model was obtained with SVM classifiers (AUC $=0.714(95 \% \mathrm{CI}, 0.602-0.827)$, external validation). Moreover, wavelet features derived from MR, PWI and DWI were associated with KRAS mutation in rectal cancer patients in [112], achieving a final AUC of 0.651 (95\% CI, 0.539-0.763) on a temporally independent validation cohort.

\subsubsection{TP-53}

One group found that an increased value of short-run low gray-level emphasis derived from the GLRLM in FDG-PET/CT was predictive for TP-53 mutation in CRC patients (AUC $=0.71$, training). Authors also reported higher heterogeneity and lower PET signal values in TP-53-mutant cases [109]. On the other hand, one study carried out with FDGPET/CT data from pancreatic ductal adenocarcinoma patients did not see a significant association between genetic alterations in TP-53 status and the radiomic features extracted from the PET images [108].

\subsubsection{HER-2}

The association of HER-2 status and CT radiomics in gastric cancer patients was investigated in [103]. Authors reported a final AUC of 0.771 (95\% CI, 0.607-0.934) on an internal validation cohort when employing a nomogram based on seven wavelet features and patient carcino-embryogenic antigen (CEA) level. One study extracted radiomic features from pre-operative MR images of patients suffering from rectal cancer, achieving a final AUC of 0.696 (95\% CI, 0.610-0.782) on a temporally independent validation cohort [112].

\subsubsection{Ki-67}

Three studies investigated the potential association of Ki-67 index and radiomic signatures [104,105,112]. A CE CT-based radiomics nomogram including six radiomic features for the gastrointestinal stromal tumors was significantly associated with Ki-67 (AUC $=0.754$, external validation) [104]. Another retrospective, multicenter study in CE CT focused on pancreatic neuroendocrine tumors showed a significant association between Ki67 and an eight-feature-combined radiomics [105]. The third study analyzed a combination of MR, PWI and DWI radiomics to predict Ki-67 expression, with a final AUC of 0.699 on a 
temporally independent cohort [112]. Different Ki-67 expression cut-off values were used on each study, ranging from 10 to $40 \%$.

\subsubsection{BRAF}

As explained in the KRAS biomarker subsection, one study investigated the relation between CE CT radiomics and the mutation signature KRAS/NRAS/BRAF together, which reported a final AUC of 0.829 on a temporally independent cohort [106].

\subsection{Liver Cancer}

\subsubsection{Summary}

Four studies were found which associated radiomics and tissue biomarkers in liver cancer patients, using either MR with contrast agents $(n=2)$ or US $(n=2)$. The most common tumor type was hepatocellular carcinoma (HCC, $n=3$ ) [113-115] followed by cholangiocarcinoma (CCA, $n=1$ ) [116]. Three tissue biomarkers were investigated: Ki-67 $(n=3)$, PD-L1 $(n=2)$ and VEGF $(n=1)$ and all were shown to be significantly correlated to radiomics ( $\mathrm{AUC}=0.85-0.97$ ). All studies employed a dataset limited to a single center; one study separated the dataset into a training and a validation cohort [116]. None of the studies reported any radiomics quality measure and only one of them was a registered prospective study [114]. A summary of the findings of this section can be found in Table 6 . 
Table 6. An overview of the radiomic studies included in the liver cancer section.

\begin{tabular}{|c|c|c|c|c|c|c|c|c|c|c|c|c|}
\hline Study & Biomarker & Alteration & Modality & Dataset Origin & Training & Validation & $\begin{array}{c}\text { Feature } \\
\text { Reduction }\end{array}$ & $\begin{array}{c}\text { Feature } \\
\text { Robustness }\end{array}$ & $\begin{array}{l}\text { \# Radiomic } \\
\text { Features }\end{array}$ & $\begin{array}{l}\text { Additional } \\
\text { Features }\end{array}$ & $\begin{array}{c}\text { Predictive Power } \\
\text { Measure = Mean }(95 \% \\
\text { Confidence Interval) }\end{array}$ & Open Source \\
\hline $\begin{array}{l}\text { Hectors et al. } \\
\quad[113]\end{array}$ & PD-L1 & expression & MRI, DWI & $\begin{array}{l}\text { Icahn School of } \\
\text { Medicine at } \\
\text { Mount Sinai, } \\
\text { New York, USA }\end{array}$ & 48 & - & no & no & 196 & $\begin{array}{l}\text { Infiltrative pattern; } \\
\text { presence of } \\
\text { multiple lesions; } \\
\text { extra-nodular } \\
\text { growth; } \\
\text { macrovascular } \\
\text { invasion; tumor } \\
\text { necrosis; tumor } \\
\text { hemorrhage; tumor } \\
\text { fat content; mosaic } \\
\text { appearance; } \\
\text { internal arteries; } \\
\text { capsule; T2 } \\
\text { hyper-intensity; } \\
\text { ADC } \\
\text { hypo-intensity; } \\
\text { wash-in/wash-out; } \\
\text { hepatobiliary phase } \\
\text { hypo-intensity; } \\
\text { ADCmin; } \\
\text { ADCmean; ER in } \\
\text { EA, LA, PV, LV and } \\
\text { hepatobiliary } \\
\text { phases; tumor size }\end{array}$ & $\begin{array}{c}\begin{array}{l}\text { Significant correlation }(p \\
<0.029)\end{array} \\
\end{array}$ & - \\
\hline Peng et al. [116] & Ki-67; VEGF & $\begin{array}{c}\text { Ki-67: High } \\
\text { expression at } \\
\geq 10 \% \\
\text { VEGF: } \\
\text { expression }\end{array}$ & US & $\begin{array}{l}\text { First Affiliated } \\
\text { Hospital of } \\
\text { Guangxi Medical } \\
\text { University, } \\
\text { Nanning, } \\
\text { Guangxi, China }\end{array}$ & $\begin{array}{l}\text { Ki-67: } \\
63 \\
\text { VEGF: } \\
39\end{array}$ & $\begin{array}{c}\text { Ki-67: } \\
27^{*} \\
\text { VEGF: } \\
18^{*}\end{array}$ & yes & no & 1,076 & - & $\begin{array}{c}\text { Ki-67: } A U C=0.848 \\
\text { Accuracy }=0.889 \\
\text { VEGF: } A U C=0.864 \\
\text { Accuracy }=0.833\end{array}$ & - \\
\hline Yao et al. [115] & Ki-67; PD-L1 & $\begin{array}{c}\text { Ki-67: High } \\
\text { expression at } \\
\geq 25 \% \\
\text { PD-L1: } \\
\text { expression }\end{array}$ & US & $\begin{array}{l}\text { Zhongshan } \\
\text { Hospital, Fudan } \\
\text { University, } \\
\text { Shanghai, China }\end{array}$ & 47 & LOOCV & yes & no & - & $\begin{array}{c}2560 \\
\text { dictionary-based } \\
\text { image features }\end{array}$ & $\begin{array}{c}\text { PD-L1: AUC }=0.97 \\
(0.89-0.98) \\
\text { Accuracy }=92 \% \\
\text { Ki-67: AUC }=0.94 \\
(0.87-0.97) \\
\text { Accuracy }=93 \%\end{array}$ & $\begin{array}{l}\text { Images on } \\
\text { request }\end{array}$ \\
\hline
\end{tabular}


Table 6. Cont.

\begin{tabular}{|c|c|c|c|c|c|c|c|c|c|c|c|c|}
\hline Study & Biomarker & Alteration & Modality & Dataset Origin & Training & Validation & $\begin{array}{c}\text { Feature } \\
\text { Reduction }\end{array}$ & $\begin{array}{c}\text { Feature } \\
\text { Robustness }\end{array}$ & $\begin{array}{c}\text { \# Radiomic } \\
\text { Features }\end{array}$ & $\begin{array}{l}\text { Additional } \\
\text { Features }\end{array}$ & $\begin{array}{c}\text { Predictive Power } \\
\text { Measure = Mean (95\% } \\
\text { Confidence Interval) }\end{array}$ & Open Source \\
\hline Ye et al. [114] & Ki-67 & $\begin{array}{l}\text { High } \\
\text { expression at } \\
\geq 15 \%\end{array}$ & MRI & $\begin{array}{l}\text { West China } \\
\text { Hospital, } \\
\text { Sichuan, China }\end{array}$ & 89 & $10-\mathrm{CV}$ & yes & no & 396 & $\begin{array}{c}\text { Serum level of } \\
\text { alpha-fetoprotein; } \\
\text { hepatitis B surface } \\
\text { antigen; hepatitis C } \\
\text { antibody; } \\
\text { Barcelona-Clinic } \\
\text { Liver Cancer } \\
\text { classification; } \\
\text { cirrhosis; } \\
\text { multifocality; } \\
\text { arterial phase } \\
\text { hyper- } \\
\text { enhancement; } \\
\text { washout, capsule } \\
\text { integrity, internal } \\
\text { arteries, tumor } \\
\text { margin, enhancing } \\
\text { capsule, } \\
\text { hepato-biliary } \\
\text { phase } \\
\text { hypo-intensity }\end{array}$ & $\begin{array}{c}\text { c-index: } 0.936 \\
(0.863-0.977)\end{array}$ & - \\
\hline
\end{tabular}

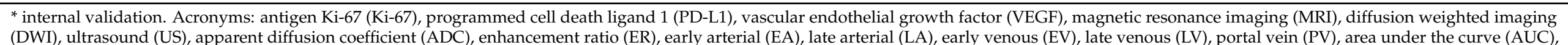
leave-one-out cross-validation (LOOCV), 10-fold cross-validation (10-CV). 


\subsubsection{PD-L1}

The best predictive performance overall for liver studies was obtained for PD-L1 in US images of HCC patients (AUC $=0.97$, cross-validation) [115]. The expression of PD-L1 was also predicted from MRI images of HCC, where the best association was found with the texture feature ADC variance. This may be interpreted as a correspondence between higher heterogeneity and higher PD-L1 expression levels [113].

\subsubsection{Ki-67}

The best AUC for Ki-67 expression prediction in HCC was obtained in [115] by means of a SVM model based on US radiomic features (AUC $=0.94$, cross-validation). Slightly worse performances (AUC $=0.804$, internal validation) were obtained with US wavelet features for CCA patients in [116]. Another group employed texture features from MR images of HCC patients [114]. Authors combined 13 features from T2W, pre-contrast (PRE), arterial phase (AP) and portal venous phase (PV) scans into a multiparametric texture signature which achieve a c-index of 0.878 after cross-validation. The features included suggested that higher intra-tumor heterogeneity correlates to higher expression of Ki-67. The latter may reflect the cell proliferation status and therefore tumor aggressiveness.

\subsubsection{VEGF}

The relationship between VEGF expression and US radiomic features was analyzed only in CCA patients [116]. Wavelet features were found to be the most relevant feature type to predict the biomarker expression (AUC $=0.864$, internal validation). These were associated with the heterogeneity of the tumor volume by the authors.

\subsection{Other Cancers}

\subsubsection{Summary}

In total, five studies were found which investigated the correlation between radiomics and molecular markers in other entities not included in the sections above: melanoma $(n=1)$ [117], thyroid cancer $(n=1)$ [118], head and neck cancer $(n=2)[119,120]$, adrenal gland carcinoma $(n=1)$ [121]. All studies showed a significant correlation between the biomarker and radiomics (AUC $=0.62-0.78$ ). None of the studies used external validation. None of the studies reported any radiomics quality measure, nor were they registered prospective studies. A summary of the findings of this section can be found in Table 7. 
Table 7. An overview of the radiomic studies included in the other cancers section.

\begin{tabular}{|c|c|c|c|c|c|c|c|c|c|c|c|c|}
\hline Study & Biomarker & Alteration & Modality & Dataset Origin & Training & Validation & $\begin{array}{c}\text { Feature } \\
\text { Reduction }\end{array}$ & $\begin{array}{c}\begin{array}{c}\text { Feature } \\
\text { Robustness }\end{array}\end{array}$ & $\begin{array}{l}\text { \# Radiomic } \\
\text { Features }\end{array}$ & $\begin{array}{c}\text { Additional } \\
\text { Features }\end{array}$ & $\begin{array}{c}\text { Predictive Power } \\
\text { Measure = Mean (95\% } \\
\text { Confidence Interval) }\end{array}$ & Open Source \\
\hline $\begin{array}{l}\text { Ahmed et al. } \\
\text { [121] }\end{array}$ & Ki-67 & $\begin{array}{c}\text { High } \\
\text { expression at } \\
\geq 10 \% \\
\text { PD-L1: High }\end{array}$ & $\mathrm{CT}$ & $\begin{array}{l}\text { MD Anderson } \\
\text { Cancer Center, } \\
\text { Texas, US }\end{array}$ & 53 & - & no & no & 106 & - & $\mathrm{AUC}=0.78$ & - \\
\hline Chen et al. [120] & $\begin{array}{c}\text { PD-L1; } \\
\text { EGFR; } \\
\text { VEGF; Ki-67 }\end{array}$ & $\begin{array}{c}\text { expression at } \\
\geq 5 \% \text { and } \geq 1 \% \\
\text { EGFR: } \\
\text { expression } \\
\text { VEGF: } \\
\text { expression } \\
\text { Ki-67: } \\
\text { expression }\end{array}$ & FDG-PET & $\begin{array}{c}\text { China Medical } \\
\text { University, } \\
\text { Taichung City, } \\
\text { Taiwan }\end{array}$ & 53 & - & no & no & 41 & $\begin{array}{l}\text { SUVmax, MTV, } \\
\text { TLGmean; smoking } \\
\text { history; tumor } \\
\text { origin; TNM stage }\end{array}$ & $\begin{array}{c}\text { PD-L1: AUC }=0.24^{1} \text {; } \\
\text { EGFR: no correlation. } \\
\text { VEGF: Correlation }(p< \\
0.05) ; \\
\text { Ki-67: Correlation }(p< \\
0.05)\end{array}$ & - \\
\hline $\begin{array}{l}\text { Saadani et al. } \\
\text { [117] }\end{array}$ & BRAF & $\begin{array}{c}\mathrm{v} 600 \mathrm{E} \\
\text { mutation }\end{array}$ & $\begin{array}{l}\text { FDG- } \\
\text { PET/CT }\end{array}$ & $\begin{array}{l}\text { Netherlands } \\
\text { Cancer Institute, } \\
\text { Amsterdam, The } \\
\text { Netherlands }\end{array}$ & 70 & $10-\mathrm{CV}$ & yes & no & 480 & $\begin{array}{l}\text { SUVmax; } \\
\text { SUVmean; } \\
\text { SUVpeak; MTV; } \\
\text { TLG; longest } \\
\text { diameter }\end{array}$ & $\mathrm{AUC}=0.62$ & - \\
\hline Yoon et al. [118] & BRAF & $\begin{array}{c}\mathrm{v} 600 \mathrm{E} \\
\text { mutation }\end{array}$ & US & $\begin{array}{c}\text { Severance } \\
\text { Hospital, Yonsei } \\
\text { University } \\
\text { College of } \\
\text { Medicine, Seoul, } \\
\text { South Korea }\end{array}$ & 387 & $140 * * *$ & yes & no & 730 & $\begin{array}{l}\text { Age; tumor size; } \\
\text { sex; }\end{array}$ & $\begin{array}{l}\mathrm{AUC}=0.629 \\
(0.516-0.742)\end{array}$ & - \\
\hline
\end{tabular}

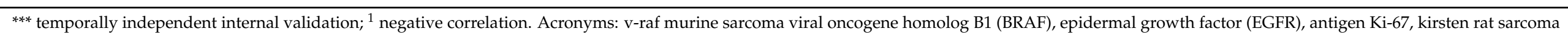

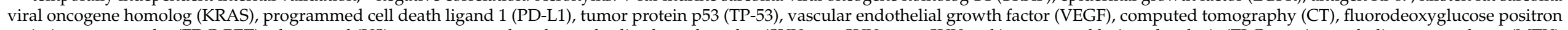

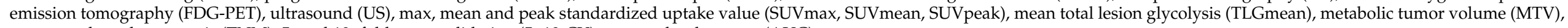
tumor, node and metastasis (TNM), 5- and 10- fold cross-validation (5-,10-CV), area under the curve (AUC). 


\subsubsection{Details}

One study explored the use of FDG-PET/CT radiomics to predict BRAFv600 mutation status in melanoma patients achieving a final AUC of 0.62 after 10-CV [117]. Another study investigated the use of US radiomics to predict BRAFv600 mutation of thyroid cancer patients with a limited predictive performance on a temporally independent validation cohort (c-statistics $=0.629)$ [118]. Two studies explored the association of different biomarkers and imaging features in head and neck squamous cell carcinoma patients. One of them reported a moderate predictive power of CT radiomics for TP-53 mutation prediction (AUC $=0.641$, 5-CV) [119], while the other study reported a limited linkage between PD-L1, VEGF, Ki-67 and EGFR expression and FDG-PET radiomics on their training cohort [120]. The latter also showed a positive correlation between PD-L1 and Ki-67 expression. The GLCM-derived feature of correlation was found to be a negative predictor of PD-L1 expression, while it was positively associated with VEGF expression. One study investigated the efficacy of CE CT radiomics to predict Ki-67 expression in adrenal gland carcinoma patients [121]. The authors reported final AUCs of $0.7-0.78$ on the training cohort after using logistic regression models based on two shape features, suggesting that high Ki-67 expression is associated with flatter and more elongated tumors.

\subsection{Feature Interpretation}

In Tables 8-10, we gathered those radiomic features employed in the best performing models for each combination of biomarker and tumor site, for MRI, CT and PET, respectively. Detailed tables including feature names and additional modalities (e.g., US or advanced MRI sequences) are shown in Supplementary Tables S1-S4. For seven studies, no interpretation was possible due to lack of information. 


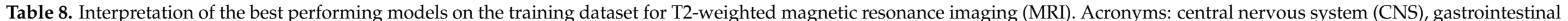

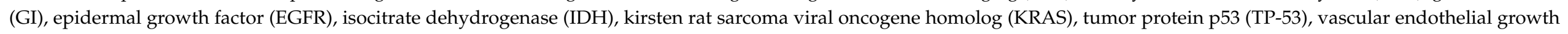
factor (VEGF).

\begin{tabular}{|c|c|c|c|c|c|c|}
\hline MRI & EGFR & Ki-67 & KRAS & TP-53 & VEGF & IDH \\
\hline CNS & $\begin{array}{c}\text { EGFR+ more } \\
\text { heterogeneous, less } \\
\text { spherical [32] }\end{array}$ & $\begin{array}{l}\text { Ki-67 high expression } \\
\text { more heterogeneous [34] }\end{array}$ & & $\begin{array}{c}\text { TP-53+ higher intensity } \\
\text { [36] }\end{array}$ & $\begin{array}{c}\text { VEGF+ more } \\
\text { heterogeneous [37] }\end{array}$ & $\begin{array}{l}\text { IDH+ more } \\
\text { homogeneous, more } \\
\text { regularly shaped [21] }\end{array}$ \\
\hline GI & & & $\begin{array}{c}\text { KRAS+ more } \\
\text { heterogeneous [110] }\end{array}$ & & & \\
\hline Liver & & $\begin{array}{c}\text { Ki-67 high expression } \\
\text { more heterogeneous [114] }\end{array}$ & & & & \\
\hline
\end{tabular}

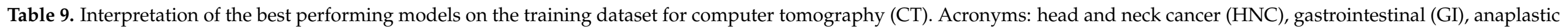

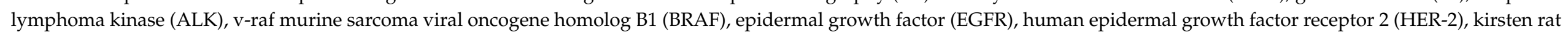
sarcoma viral oncogene homolog (KRAS), programmed cell death ligand 1 (PD-L1), tumor protein p53 (TP-53).

\begin{tabular}{|c|c|c|c|c|c|c|c|}
\hline $\mathrm{CT}$ & EGFR & Ki-67 & KRAS/BRAF & TP-53 & HER-2 & ALK & PD-L1 \\
\hline HNC & & $\begin{array}{c}\text { Ki-67 high expression } \\
\text { more heterogeneous [120] }\end{array}$ & & $\begin{array}{c}\text { TP-53+ more } \\
\text { heterogeneous [119] }\end{array}$ & & & \\
\hline Lung & $\begin{array}{l}\text { EGFR+ more } \\
\text { heterogeneous, } \\
\text { smaller [83] }\end{array}$ & $\begin{array}{l}\text { Ki-67 high expression } \\
\text { more homogeneous, more } \\
\text { elongated [90] }\end{array}$ & $\begin{array}{c}\text { KRAS+ more } \\
\text { homogeneous [83] }\end{array}$ & & & $\begin{array}{c}\text { ALK+ higher density } \\
\text { [87] }\end{array}$ & $\begin{array}{c}\text { PD-L1+ more } \\
\text { homogeneous [89] }\end{array}$ \\
\hline GI & & $\begin{array}{l}\text { Ki-67 high expression } \\
\text { more heterogeneous [104] }\end{array}$ & $\begin{array}{l}\text { KRAS/BRAF+ more } \\
\text { heterogeneous [106] }\end{array}$ & & $\begin{array}{c}\text { HER-2+ more } \\
\text { heterogeneous [103] }\end{array}$ & & \\
\hline
\end{tabular}




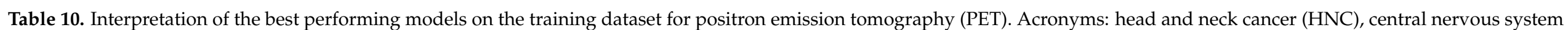

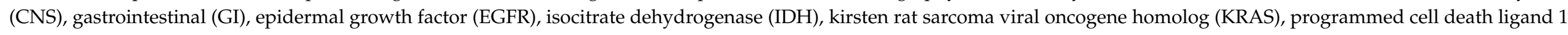
(PD-L1), tumor protein p53 (TP-53), vascular endothelial growth factor (VEGF).

\begin{tabular}{|c|c|c|c|c|c|c|c|}
\hline PET & EGFR & Ki-67 & KRAS & TP-53 & VEGF & IDH & PD-L1 \\
\hline CNS & & & & & & $\begin{array}{c}\text { IDH+ more } \\
\text { homogeneous, less } \\
\text { spherical [50] }\end{array}$ & \\
\hline HNC & & & & & $\begin{array}{c}\text { VEGF+ more } \\
\text { heterogeneous [120] }\end{array}$ & & $\begin{array}{c}\text { PD-L1+ more } \\
\text { heterogeneous [120] }\end{array}$ \\
\hline Lung & $\begin{array}{c}\text { EGFR+ more } \\
\text { heterogeneous, more } \\
\text { compact [100] }\end{array}$ & & & & & & \\
\hline GI & & & $\begin{array}{l}\text { KRAS+ lower } \\
\text { intensity [108] }\end{array}$ & $\begin{array}{c}\text { TP-53+ more } \\
\text { heterogeneous [109] }\end{array}$ & & & \\
\hline $\begin{array}{l}\text { Adrenal gland } \\
\text { carcinoma }\end{array}$ & & $\begin{array}{c}\text { Ki-67 high expression } \\
\text { more elongated and } \\
\text { flatter [121] }\end{array}$ & & & & & \\
\hline
\end{tabular}


Oftentimes, dysregulation of one specific biomarker led to similar tumor phenotype across entities and imaging modalities. This was the case for EGFR-mutant tumors, which exhibited greater textural heterogeneity in CNS MRI, PWI and DWI, as well as in lung CT and PET. Similarly, alteration of TP-53 status was associated with increased heterogeneity in CT of HN and PET of colorectal cancer. IDH-mutant tumors were reported to have greater textural homogeneity in MRI, DWI, PWI, DKI and FDG-PET in CNS. High Ki-67expressing tumors were reported to be more homogenous in CT for lung cancer but more heterogeneous for gynecological tumors and head and neck tumors. KRAS+ was shown to be more homogeneous for $\mathrm{CT}$ in lung, but more heterogeneous for gastrointestinal cancer.

\subsection{Results per Biomarker}

An overview of the analyzed studies per biomarker can be found in Tables S5-S14 in the Supplementary Materials.

\section{Discussion}

In recent decades, extensive genomic studies have leveraged our understanding of cancer biology and pathophysiology. The identification of key genetic alterations that drive oncogenesis and their subsequent molecular markers has led to a more accurate and comprehensive patient-specific treatment planning and adaptation [2]. Furthermore, the field of radiomics, i.e., the quantitative, high-throughput analysis of medical images, has emerged as a potential diagnostic, prognostic and predictive tool in clinical decisionsupport systems. This is of particular interest in cancer treatment, where medical imaging is routinely performed with diagnostic and monitoring purposes. Nonetheless, the reliability, clinical applicability and biological meaning of radiomics models and imaging biomarkers has to be extensively validated before they can be incorporated into clinical routine [17]. Hence, the primary objective of this review was to identify key radiomic features associated with specific tumor molecular markers through an electronic search of peer-reviewed journal publications.

For this purpose, we limited our search to ten cancer biological endpoints commonly investigated and used in clinical practice, which apply to a broad range of cancer types. Other, even though valid, biomarkers, such as methylation status or indicators for virusborn cancers were deliberately excluded as their origin and/or mechanism leading to malignant transformation of healthy cells is not trivially comparable. Other examples of biomarkers excluded in this review are the loss of tumor suppressors in cancer such as breast cancer genes 1 and 2 ( $B R C A-1, B R C A-2)$, RNAs, proteins such as prostate-specific antigen (PSA) or circulating tumor DNA (ct-DNA) [1]. By focusing on this compact set of biomarkers, we aimed to summarize the reported associations between radiomics and signature molecules and eventually contribute to the promotion of radiomics as a valid diagnostic, prognostic and predictive tool in cancer treatment. We are aware that the selection of biomarkers is not complete but due to the sheer number of biomarkers and the variability thereof, the search had to be narrowed in order to perform a meaningful systematic review.

Most of the studies included in this review reported some association between the selected biomarkers and radiomics, suggesting that mutated and non-mutated tumors have different growth patterns that are identifiable in high-throughput imaging. The association of textural, intensity, shape, size and wavelet image features with tumor biomarkers entails an advance in feature interpretability, as shown in Tables $8-10$, which brings radiomics closer to its application in a clinical setting.

In total, 96 out of 104 studies found a significant relationship between at least one of the studied biomarkers and one or more radiomic features. However, only 7 studies validated their models on external cohorts, 11 studies on temporally independent cohorts and 14 studies did not use any form of validation. Additionally, only 7 out of 104 included a prospectively collected dataset, which is necessary to confirm the clinical validity and usefulness of any radiomics signature. Along these lines, we believe greater effort should 
be made to employ larger, multi-institutional cohorts, either by means of new data-sharing agreements among research groups or through distributed learning. The feasibility of the latter has already been shown in a number of studies and entails new possibilities for training reliable radiomics models [122,123]. Furthermore, only 37 studies performed some type of robustness analysis of the selected features. Different image acquisition parameters, scanner models, pre-processing and region of interest segmentation techniques among other factors have been shown to significantly affect feature robustness and results reproducibility, and should be evaluated in greater detail [124-126]. Moreover, we would like to encourage projects such as the image biomarker standardization initiative (IBSI) [127], which works towards the homogenization of image feature extraction and analysis.

Another factor that hindered results interpretation and studies comparison was the great variability in biomarker expression levels employed as cut-offs to stratify patients. Currently, there exist a lack of standardization of immunohistochemistry techniques for biomarker staining and scoring systems, leading to moderate intra/inter-laboratory and intra/inter-observer variabilities $[1,128]$. This could potentially explain the observed phenotype disagreement across different entities and modalities for Ki-67, PD-L1 and KRAS biomarkers, as described in Tables 8-10. However, as previously explained, it should be noted that these studies were included on the interpretation table based on their performance on the training set, and, for the vast majority, external validation remains to be accomplished.

In an attempt to standardize the clinical utility evaluation of radiomics studies, as well as to increase transparency and minimize risk of bias, two rigorous reporting guidelines, the TRIPOD [17] and the RQS [4] scores, have been devised. In Tables 2-7, we gathered some of the most relevant reporting criteria such as the type of validation used, the performance of feature reduction and robustness analysis, the use of discrimination statistics, the inclusion of non-radiomic features and the public availability of the code and/or data. However, none of the studies included in this review followed explicitly TRIPOD or RQS guidelines. We would like to encourage the use of such guidelines as they provide a common framework to compare state-of-the-art results in radiomics and bring closer its incorporation into clinical decision support-systems.

\section{Conclusions}

In summary, radiomics from different modalities and cancer entities is a promising tool for tumor biology assessment. Nevertheless, a large majority of studies included in this review only employed internal validation datasets or bootstrap and cross-validation techniques to assess model performance. Thus, further multi-center, prospective studies are required to validate the reported outcomes. Moreover, none of the studies followed any reporting or quality assurance protocols. Hence, we would like to encourage the employment of reporting guidelines such as TRIPOD and RQS scores, as well as the use of IBSI-standardized radiomics software. As a closing remark, we would like to emphasize the utmost importance of transparency to ensure the reproducibility of radiomics studies.

Supplementary Materials: The following are available online at https: / www.mdpi.com/article / 10.3390/cancers13123015/s1, List S1: Queries employed in the PubMed search, List S2. PRISMA Checklist, Table S1: Feature interpretation for ALK, BRAF and EGFR, Table S2: Feature interpretation for HER-2, IDH and Ki-67, Table S3: Feature interpretation for KRAS, KRAS/BRAF and PD-L1, Table S4: Feature interpretation for TP-53 and VEGF, Table S5: An overview of the radiomic studies included for IDH biomarker, Table S6: An overview of the radiomic studies included for EGFR biomarker, Table S7: An overview of the radiomic studies included for VEGF biomarker, Table S8: An overview of the radiomic studies included for HER-2 biomarker, Table S9: An overview of the radiomic studies included for ALK biomarker, Table S10: An overview of the radiomic studies included for BRAF biomarker, Table S11: An overview of the radiomic studies included for PD-L1 biomarker, Table S12: An overview of the radiomic studies included for TP-53 biomarker, Table S13: An overview of the radiomic studies included for KRAS biomarker and Table S14: An overview of the radiomic studies included for Ki-67 biomarker. 
Author Contributions: The processes of screening, eligibility evaluation and extraction of data for the meta-analysis were performed independently by the authors A.L.G.S.-E., D.V., F.T., R.D.B. and V.W. The authors J.E.v.T., S.T.-L., M.P. and M.G. supervised the process. All authors contributed to writing and reviewing the manuscript. All authors have read and agreed to the published version of the manuscript.

Funding: This work was partially supported by the Swiss National Science Foundation (310030_173303, 310030_172885 and CRSII5_183478), the Klinischer Forschungsschwerpunkt (KFSP) Artificial Intelligence in Oncological Imaging from the University of Zurich and the Swiss Personalized Health Network (SPHN) IMAGINE.

Institutional Review Board Statement: Not applicable.

Informed Consent Statement: Not applicable.

Conflicts of Interest: The authors declare no conflict of interest.

\section{References}

1. Goossens, N.; Nakagawa, S.; Sun, X.; Hoshida, Y. Cancer biomarker discovery and validation. Transl. Cancer Res. 2015, 4, 256-269.

2. Malone, E.R.; Oliva, M.; Sabatini, P.J.B.; Stockley, T.L.; Siu, L.L. Molecular profiling for precision cancer therapies. Genome Med. 2020, 12, 8. [CrossRef]

3. Song, J.; Yin, Y.; Wang, H.; Chang, Z.; Liu, Z.; Cui, L. A review of original articles published in the emerging field of radiomics. Eur. J. Radiol. 2020, 127, 108991. [CrossRef]

4. $\quad$ Lambin, P.; Leijenaar, R.T.H.; Deist, T.M.; Peerlings, J.; de Jong, E.E.C.; van Timmeren, J.; Sanduleanu, S.; Larue, R.; Even, A.J.G.; Jochems, A.; et al. Radiomics: The bridge between medical imaging and personalized medicine. Nat. Rev. Clin. Oncol. 2017, 14, 749-762. [CrossRef]

5. Bublil, E.M.; Yarden, Y. The EGF receptor family: Spearheading a merger of signaling and therapeutics. Curr. Opin. Cell Biol. 2007, 19, 124-134. [CrossRef] [PubMed]

6. García-Regalado, A.; La Rosa, C.H.G.-D. The Role of Anaplastic Lymphoma Kinase in Human Cancers. Oncol. Hematol. Rev. 2013, 9, 149-153. [CrossRef]

7. Zaman, A.; Wu, W.; Bivona, T.G. Targeting Oncogenic BRAF: Past, Present, and Future. Cancers 2019, 11, 1197. [CrossRef]

8. Liu, P.; Wang, Y.; Li, X. Targeting the untargetable KRAS in cancer therapy. Acta Pharm. Sin. B 2019, 9, 871-879. [CrossRef] [PubMed]

9. Neufeld, G.; Cohen, T.; Gengrinovitch, S.; Poltorak, Z. Vascular endothelial growth factor (VEGF) and its receptors. FASEB J. 1999, 13, 9-22. [CrossRef]

10. Read, A.; Strachan, T. Chapter 18: Cancer Genetics. Human Molecular Genetics, 2nd ed.; Wiley: New York, NY, USA, 1999.

11. Salmaninejad, A.; Valilou, S.F.; Shabgah, A.G.; Aslani, S.; Alimardani, M.; Pasdar, A.; Sahebkar, A. PD-1/PD-L1 pathway: Basic biology and role in cancer immunotherapy. J. Cell. Physiol. 2019, 234, 16824-16837. [CrossRef]

12. Bleeker, F.E.; Molenaar, R.J.; Leenstra, S. Recent advances in the molecular understanding of glioblastoma. J. Neuro-Oncol. 2012, 108, 11-27. [CrossRef]

13. Scholzen, T.; Gerdes, J. The Ki-67 protein: From the known and the unknown. J. Cell. Physiol. 2000, 182, 311-322. [CrossRef]

14. Biomarkers in Risk Assessment: Validity and Validation (EHC 222, 2001). Available online: http:/ / www.inchem.org/documents/ ehc/ehc/ehc222.htm (accessed on 5 June 2021).

15. Moher, D.; Shamseer, L.; Clarke, M.; Ghersi, D.; Liberati, A.; Petticrew, M.; Shekelle, P.; Stewart, L.A.; PRISMA-P Group. Preferred reporting items for systematic review and meta-analysis protocols (PRISMA-P) 2015 statement. Syst. Rev. 2015, 4, 1. [CrossRef] [PubMed]

16. Zotero I Your Personal Research Assistant. Available online: http:/ /www.zotero.org (accessed on 29 September 2020).

17. Tripod Statement. Available online: https://www.tripod-statement.org/ (accessed on 6 June 2021).

18. Wu, G.; Chen, Y.; Wang, Y.; Yu, J.; Lv, X.; Ju, X.; Shi, Z.; Chen, L.; Chen, Z. Sparse Representation-Based Radiomics for the Diagnosis of Brain Tumors. IEEE Trans. Med. Imaging 2018, 37, 893-905. [CrossRef] [PubMed]

19. Li, Z.-C.; Bai, H.; Sun, Q.; Zhao, Y.; Lv, Y.; Zhou, J.; Liang, C.; Chen, Y.; Liang, D.; Zheng, H. Multiregional radiomics profiling from multiparametric MRI: Identifying an imaging predictor of IDH1 mutation status in glioblastoma. Cancer Med. 2018, 7, 5999-6009. [CrossRef] [PubMed]

20. Choi, Y.; Nam, Y.; Lee, Y.S.; Kim, J.; Ahn, K.-J.; Jang, J.; Shin, N.-Y.; Kim, B.-S.; Jeon, S.-S. IDH1 mutation prediction using MR-based radiomics in glioblastoma: Comparison between manual and fully automated deep learning-based approach of tumor segmentation. Eur. J. Radiol. 2020, 128, 109031. [CrossRef] [PubMed]

21. Liu, X.; Li, Y.; Li, S.; Fan, X.; Sun, Z.; Yang, Z.; Wang, K.; Zhang, Z.; Jiang, T.; Liu, Y.; et al. IDH mutation-specific radiomic signature in lower-grade gliomas. Aging 2019, 11, 673-696. [CrossRef]

22. Li, Z.; Wang, Y.; Yu, J.; Guo, Y.; Cao, W. Deep Learning based Radiomics (DLR) and its usage in noninvasive IDH1 prediction for low grade glioma. Sci. Rep. 2017, 7, 5467. [CrossRef] 
23. Yu, J.; Shi, Z.; Lian, Y.; Li, Z.; Liu, T.; Gao, Y.; Wang, Y.; Chen, L.; Mao, Y. Noninvasive IDH1 mutation estimation based on a quantitative radiomics approach for grade II glioma. Eur. Radiol. 2017, 27, 3509-3522. [CrossRef]

24. Yu, J.; Shi, Z.; Ji, C.; Lian, Y.; Wang, Y.; Chen, L.; Mao, Y. Anatomical location differences between mutated and wild-type isocitrate dehydrogenase 1 in low-grade gliomas. Int. J. Neurosci. 2017, 127, 873-880. [CrossRef]

25. Liu, T.; Wu, G.; Yu, J.; Guo, Y.; Wang, Y.; Shi, Z.; Chen, L. A mRMRMSRC feature selection method for radiomics approach. In Proceedings of the 2017 39th Annual International Conference of the IEEE Engineering in Medicine and Biology Society (EMBC), Jeju, Korea, 11-15 July 2017; Volume 2017, pp. 616-619. [CrossRef]

26. Arita, H.; Kinoshita, M.; Kawaguchi, A.; Takahashi, M.; Narita, Y.; Terakawa, Y.; Tsuyuguchi, N.; Okita, Y.; Nonaka, M.; Moriuchi, S.; et al. Lesion location implemented magnetic resonance imaging radiomics for predicting IDH and TERT promoter mutations in grade II/III gliomas. Sci. Rep. 2018, 8, 11773. [CrossRef] [PubMed]

27. Fukuma, R.; Yanagisawa, T.; Kinoshita, M.; Shinozaki, T.; Arita, H.; Kawaguchi, A.; Takahashi, M.; Narita, Y.; Terakawa, Y.; Tsuyuguchi, N.; et al. Prediction of IDH and TERT promoter mutations in low-grade glioma from magnetic resonance images using a convolutional neural network. Sci. Rep. 2019, 9, 20311. [CrossRef] [PubMed]

28. Kuthuru, S.; Deaderick, W.; Bai, H.; Su, C.; Vu, T.; Monga, V.; Rao, A. A Visually Interpretable, Dictionary-Based Approach to Imaging-Genomic Modeling, with Low-Grade Glioma as a Case Study. Cancer Inform. 2018, 17, 1176935118802796. [CrossRef]

29. Zhou, H.; Vallières, M.; Bai, H.X.; Su, C.; Tang, H.; Oldridge, D.; Zhang, Z.; Xiao, B.; Liao, W.; Tao, Y.; et al. MRI features predict survival and molecular markers in diffuse lower-grade gliomas. Neuro-Oncology 2017, 19, 862-870. [CrossRef] [PubMed]

30. Zhang, X.; Tian, Q.; Wang, L.; Liu, Y.; Li, B.; Liang, Z.; Gao, P.; Zheng, K.; Zhao, B.; Lu, H. Radiomics Strategy for Molecular Subtype Stratification of Lower-Grade Glioma: Detecting IDH andTP53Mutations Based on Multimodal MRI. J. Magn. Reson. Imaging 2018, 48, 916-926. [CrossRef]

31. Lee, J.; Narang, S.; Martinez, J.J.; Rao, G.; Rao, A. Associating spatial diversity features of radiologically defined tumor habitats with epidermal growth factor receptor driver status and 12-month survival in glioblastoma: Methods and preliminary investigation. J. Med. Imaging 2015, 2, 041006. [CrossRef]

32. Li, Y.; Liu, X.; Xu, K.; Qian, Z.; Wang, K.; Fan, X.; Li, S.; Wang, Y.; Jiang, T. MRI features can predict EGFR expression in lower grade gliomas: A voxel-based radiomic analysis. Eur. Radiol. 2018, 28, 356-362. [CrossRef]

33. Li, J.; Liu, S.; Qin, Y.; Zhang, Y.; Wang, N.; Liu, H. High-order radiomics features based on T2 FLAIR MRI predict multiple glioma immunohistochemical features: A more precise and personalized gliomas management. PLoS ONE 2020, 15, e0227703. [CrossRef]

34. Li, Y.; Qian, Z.; Xu, K.; Wang, K.; Fan, X.; Li, S.; Liu, X.; Wang, Y.; Jiang, T. Radiomic features predict Ki-67 expression level and survival in lower grade gliomas. J. Neuro-Oncol. 2017, 135, 317-324. [CrossRef]

35. Ugga, L.; Cuocolo, R.; Solari, D.; Guadagno, E.; D’Amico, A.; Somma, T.; Cappabianca, P.; Caro, M.L.D.B.D.; Cavallo, L.M.; Brunetti, A. Prediction of high proliferative index in pituitary macroadenomas using MRI-based radiomics and machine learning. Neuroradiology 2019, 61, 1365-1373. [CrossRef]

36. Li, Y.; Qian, Z.; Xu, K.; Wang, K.; Fan, X.; Li, S.; Jiang, T.; Liu, X.; Wang, Y. MRI features predict p53 status in lower-grade gliomas via a machine-learning approach. NeuroImage Clin. 2018, 17, 306-311. [CrossRef] [PubMed]

37. Sun, Z.; Li, Y.; Wang, Y.; Fan, X.; Xu, K.; Wang, K.; Li, S.; Zhang, Z.; Jiang, T.; Liu, X. Radiogenomic analysis of vascular endothelial growth factor in patients with diffuse gliomas. Cancer Imaging 2019, 19, 68. [CrossRef] [PubMed]

38. Wu, S.; Meng, J.; Yu, Q.; Li, P.; Fu, S. Radiomics-based machine learning methods for isocitrate dehydrogenase genotype prediction of diffuse gliomas. J. Cancer Res. Clin. Oncol. 2019, 145, 543-550. [CrossRef]

39. Binder, Z.A.; Thorne, A.H.; Bakas, S.; Wileyto, E.P.; Bilello, M.; Akbari, H.; Rathore, S.; Ha, S.M.; Zhang, L.; Ferguson, C.J.; et al. Epidermal Growth Factor Receptor Extracellular Domain Mutations in Glioblastoma Present Opportunities for Clinical Imaging and Therapeutic Development. Cancer Cell 2018, 34, 163-177.e7. [CrossRef] [PubMed]

40. Rathore, S.; Akbari, H.; Rozycki, M.; Abdullah, K.G.; Nasrallah, M.P.; Binder, Z.A.; Davuluri, R.V.; Lustig, R.A.; Dahmane, N.; Bilello, M.; et al. Radiomic MRI signature reveals three distinct subtypes of glioblastoma with different clinical and molecular characteristics, offering prognostic value beyond IDH1. Sci. Rep. 2018, 8, 5087. [CrossRef] [PubMed]

41. Akbari, H.; Bakas, S.; Pisapia, J.M.; Nasrallah, M.P.; Rozycki, M.; Martinez-Lage, M.; Morrissette, J.J.D.; Dahmane, N.; O’Rourke, D.M.; Davatzikos, C. In vivoevaluation of EGFRvIII mutation in primary glioblastoma patients via complex multiparametric MRI signature. Neuro-Oncology 2018, 20, 1068-1079. [CrossRef]

42. Tan, Y.; Zhang, S.-T.; Wei, J.-W.; Dong, D.; Wang, X.-C.; Yang, G.-Q.; Tian, J.; Zhang, H. A radiomics nomogram may improve the prediction of IDH genotype for astrocytoma before surgery. Eur. Radiol. 2019, 29, 3325-3337. [CrossRef]

43. Lu, C.-F.; Hsu, F.-T.; Hsieh, K.L.-C.; Kao, Y.-C.J.; Cheng, S.-J.; Hsu, J.B.-K.; Tsai, P.-H.; Chen, R.-J.; Huang, C.-C.; Yen, Y.; et al. Machine Learning-Based Radiomics for Molecular Subtyping of Gliomas. Clin. Cancer Res. 2018, 24, 4429-4436. [CrossRef]

44. Su, C.; Jiang, J.; Zhang, S.; Shi, J.; Xu, K.; Shen, N.; Zhang, J.; Li, L.; Zhao, L.; Zhang, J.; et al. Radiomics based on multicontrast MRI can precisely differentiate among glioma subtypes and predict tumour-proliferative behaviour. Eur. Radiol. 2019, 29, 1986-1996. [CrossRef]

45. Tan, Y.; Mu, W.; Wang, X.-C.; Yang, G.-Q.; Gillies, R.J.; Zhang, H. Whole-tumor radiomics analysis of DKI and DTI may improve the prediction of genotypes for astrocytomas: A preliminary study. Eur. J. Radiol. 2020, 124, 108785. [CrossRef]

46. Park, C.J.; Choi, Y.S.; Park, Y.W.; Ahn, S.S.; Kang, S.-G.; Chang, J.-H.; Kim, S.H.; Lee, S.-K. Diffusion tensor imaging radiomics in lower-grade glioma: Improving subtyping of isocitrate dehydrogenase mutation status. Neuroradiology 2020, 62, 319-326. [CrossRef] 
47. Kim, M.; Jung, S.Y.; Park, J.E.; Jo, Y.; Park, S.Y.; Nam, S.J.; Kim, J.H.; Kim, H.S. Diffusion- and perfusion-weighted MRI radiomics model may predict isocitrate dehydrogenase (IDH) mutation and tumor aggressiveness in diffuse lower grade glioma. Eur. Radiol. 2020, 30, 2142-2151. [CrossRef]

48. Lee, M.H.; Kim, J.; Kim, S.-T.; Shin, H.-M.; You, H.-J.; Choi, J.W.; Seol, H.J.; Nam, D.-H.; Lee, J.-I.; Kong, D.-S. Prediction of IDH1 Mutation Status in Glioblastoma Using Machine Learning Technique Based on Quantitative Radiomic Data. World Neurosurg. 2019, 125, e688-e696. [CrossRef] [PubMed]

49. Ren, Y.; Zhang, X.; Rui, W.; Pang, H.; Qiu, T.; Wang, J.; Xie, Q.; Jin, T.; Zhang, H.; Chen, H.; et al. Noninvasive Prediction of IDH1 Mutation and ATRX Expression Loss in Low-Grade Gliomas Using Multiparametric MR Radiomic Features. J. Magn. Reson. Imaging 2019, 49, 808-817. [CrossRef] [PubMed]

50. Li, L.; Mu, W.; Wang, Y.; Liu, Z.; Liu, Z.; Wang, Y.; Ma, W.; Kong, Z.; Wang, S.; Zhou, X.; et al. A Non-invasive Radiomic Method Using 18F-FDG PET Predicts Isocitrate Dehydrogenase Genotype and Prognosis in Patients with Glioma. Front. Oncol. 2019, 9 , 1183. [CrossRef]

51. Kong, Z.; Li, J.; Liu, Z.; Liu, Z.; Zhao, D.; Cheng, X.; Li, L.; Lin, Y.; Wang, Y.; Tian, J.; et al. Radiomics signature based on FDG-PET predicts proliferative activity in primary glioma. Clin. Radiol. 2019, 74, 815.e15-815.e23. [CrossRef] [PubMed]

52. Lohmann, P.; Lerche, C.; Bauer, E.K.; Steger, J.; Stoffels, G.; Blau, T.; Dunkl, V.; Kocher, M.; Viswanathan, S.; Filss, C.P.; et al. Predicting IDH genotype in gliomas using FET PET radiomics. Sci. Rep. 2018, 8, 13328. [CrossRef]

53. Han, Y.; Wang, W.; Yang, Y.; Sun, Y.-Z.; Xiao, G.; Tian, Q.; Zhang, J.; Cui, G.-B.; Yan, L.-F. Amide Proton Transfer Imaging in Predicting Isocitrate Dehydrogenase 1 Mutation Status of Grade II/III Gliomas Based on Support Vector Machine. Front. Neurosci. 2020, 14, 144. [CrossRef] [PubMed]

54. Li, H.; Zhu, Y.; Burnside, E.S.; Huang, E.; Drukker, K.; Hoadley, K.A.; Fan, C.; Conzen, S.D.; Zuley, M.; Net, J.M.; et al. Quantitative MRI radiomics in the prediction of molecular classifications of breast cancer subtypes in the TCGA/TCIA data set. NPJ Breast Cancer 2016, 2, 16012. [CrossRef]

55. Lin, P.; Liu, W.; Li, X.; Wan, D.; Qin, H.; Li, Q.; Chen, G.; He, Y.; Yang, H. MRI-based radiogenomics analysis for predicting genetic alterations in oncogenic signalling pathways in invasive breast carcinoma. Clin. Radiol. 2020, 75, 561.e1-561.e11. [CrossRef]

56. Ma, W.; Ji, Y.; Qi, L.; Guo, X.; Jian, X.; Liu, P. Breast cancer Ki67 expression prediction by DCE-MRI radiomics features. Clin. Radiol. 2018, 73, 909.e1-909.e5. [CrossRef]

57. Monti, S.; Aiello, M.; Incoronato, M.; Grimaldi, A.M.; Moscarino, M.; Mirabelli, P.; Ferbo, U.; Cavaliere, C.; Salvatore, M. DCE-MRI Pharmacokinetic-Based Phenotyping of Invasive Ductal Carcinoma: A Radiomic Study for Prediction of Histological Outcomes. Contrast Media Mol. Imaging 2018, 2018, 5076269. [CrossRef] [PubMed]

58. Li, W.; Yu, K.; Feng, C.; Zhao, D. Molecular Subtypes Recognition of Breast Cancer in Dynamic Contrast-Enhanced Breast Magnetic Resonance Imaging Phenotypes from Radiomics Data. Comput. Math. Methods Med. 2019, 2019, 6978650. [CrossRef] [PubMed]

59. Fan, M.; Li, H.; Wang, S.; Zheng, B.; Zhang, J.; Li, L. Radiomic analysis reveals DCE-MRI features for prediction of molecular subtypes of breast cancer. PLoS ONE 2017, 12, e0171683. [CrossRef] [PubMed]

60. Castaldo, R.; Pane, K.; Nicolai, E.; Salvatore, M.; Franzese, M. The Impact of Normalization Approaches to Automatically Detect Radiogenomic Phenotypes Characterizing Breast Cancer Receptors Status. Cancers 2020, 12, 518. [CrossRef]

61. Braman, N.; Prasanna, P.; Whitney, J.; Singh, S.; Beig, N.; Etesami, M.; Bates, D.D.B.; Gallagher, K.; Bloch, B.N.; Vulchi, M.; et al. Association of Peritumoral Radiomics with Tumor Biology and Pathologic Response to Preoperative Targeted Therapy for HER2 (ERBB2)-Positive Breast Cancer. JAMA Netw. Open 2019, 2, e192561. [CrossRef] [PubMed]

62. Zhou, X.; Gao, F.; Duan, S.; Zhang, L.; Liu, Y.; Zhou, J.; Bai, G.; Tao, W. Radiomic features of Pk-DCE MRI parameters based on the extensive Tofts model in application of breast cancer. Phys. Eng. Sci. Med. 2020, 43, 517-524. [CrossRef] [PubMed]

63. Liang, C.; Cheng, Z.; Huang, Y.; He, L.; Chen, X.; Ma, Z.; Huang, X.; Liang, C.; Liu, Z. An MRI-based Radiomics Classifier for Preoperative Prediction of Ki-67 Status in Breast Cancer. Acad. Radiol. 2018, 25, 1111-1117. [CrossRef]

64. Leithner, D.; Horvat, J.V.; Marino, M.A.; Bernard-Davila, B.; Jochelson, M.S.; Ochoa-Albiztegui, R.E.; Martinez, D.; Morris, E.A.; Thakur, S.; Pinker, K. Radiomic signatures with contrast-enhanced magnetic resonance imaging for the assessment of breast cancer receptor status and molecular subtypes: Initial results. Breast Cancer Res. 2019, 21, 106. [CrossRef]

65. Zhang, Y.; Zhu, Y.; Zhang, K.; Liu, Y.; Cui, J.; Tao, J.; Wang, Y.; Wang, S. Invasive ductal breast cancer: Preoperative predict Ki-67 index based on radiomics of ADC maps. Radiol. Med. 2020, 125, 109-116. [CrossRef]

66. Fan, M.; Yuan, W.; Zhao, W.; Xu, M.; Wang, S.; Gao, X.; Li, L. Joint Prediction of Breast Cancer Histological Grade and Ki-67 Expression Level Based on DCE-MRI and DWI Radiomics. IEEE J. Biomed. Health Inform. 2020, 24, 1632-1642. [CrossRef]

67. Tagliafico, A.S.; Bignotti, B.; Rossi, F.; Matos, J.; Calabrese, M.; Valdora, F.; Houssami, N. Breast cancer Ki-67 expression prediction by digital breast tomosynthesis radiomics features. Eur. Radiol. Exp. 2019, 3, 36. [CrossRef]

68. Antunovic, L.; Gallivanone, F.; Sollini, M.; Sagona, A.; Invento, A.; Manfrinato, G.; Kirienko, M.; Tinterri, C.; Chiti, A.; Castiglioni, I. [18F]FDG PET/CT features for the molecular characterization of primary breast tumors. Eur. J. Nucl. Med. Mol. Imaging 2017, 44, 1945-1954. [CrossRef]

69. Zhou, J.; Tan, H.; Bai, Y.; Li, J.; Lu, Q.; Chen, R.; Zhang, M.; Feng, Q.; Wang, M. Evaluating the HER-2 status of breast cancer using mammography radiomics features. Eur. J. Radiol. 2019, 121, 108718. [CrossRef]

70. Aerts, H.J.W.L.; Grossmann, P.; Tan, Y.; Oxnard, G.R.; Rizvi, N.; Schwartz, L.H.; Zhao, B. Defining a Radiomic Response Phenotype: A Pilot Study using targeted therapy in NSCLC. Sci. Rep. 2016, 6, 33860. [CrossRef] [PubMed] 
71. Huang, Q.; Lu, L.; Dercle, L.; Lichtenstein, P.; Li, Y.; Yin, Q.; Zong, M.; Schwartz, L.; Zhao, B. Interobserver variability in tumor contouring affects the use of radiomics to predict mutational status. J. Med. Imaging 2018, 5, 011005. [CrossRef] [PubMed]

72. Jia, T.-Y.; Xiong, J.-F.; Li, X.-Y.; Yu, W.; Xu, Z.-Y.; Cai, X.-W.; Ma, J.-C.; Ren, Y.-C.; Larsson, R.; Zhang, J.; et al. Identifying EGFR mutations in lung adenocarcinoma by noninvasive imaging using radiomics features and random forest modeling. Eur. Radiol. 2019, 29, 4742-4750. [CrossRef] [PubMed]

73. Lu, X.; Li, M.; Zhang, H.-M.; Hua, S.; Meng, F.; Yang, H.; Li, X.; Cao, D. A novel radiomic nomogram for predicting epidermal growth factor receptor mutation in peripheral lung adenocarcinoma. Phys. Med. Biol. 2020, 65, 055012. [CrossRef] [PubMed]

74. Li, Y.; Lu, L.; Xiao, M.; Dercle, L.; Huang, Y.; Zhang, Z.; Schwartz, L.H.; Li, D.; Zhao, B. CT Slice Thickness and Convolution Kernel Affect Performance of a Radiomic Model for Predicting EGFR Status in Non-Small Cell Lung Cancer: A Preliminary Study. Sci. Rep. 2018, 8, 17913. [CrossRef]

75. Li, X.-Y.; Xiong, J.-F.; Jia, T.-Y.; Shen, T.-L.; Hou, R.-P.; Zhao, J.; Fu, X.-L. Detection of epithelial growth factor receptor (EGFR) mutations on CT images of patients with lung adenocarcinoma using radiomics and/or multi-level residual convolutionary neural networks. J. Thorac. Dis. 2018, 10, 6624-6635. [CrossRef] [PubMed]

76. Li, S.; Ding, C.; Zhang, H.; Song, J.; Wu, L. Radiomics for the prediction of EGFR mutation subtypes in non-small cell lung cancer. Med. Phys. 2019, 46, 4545-4552. [CrossRef]

77. Mei, D.; Luo, Y.; Wang, Y.; Gong, J. CT texture analysis of lung adenocarcinoma: Can Radiomic features be surrogate biomarkers for EGFR mutation statuses. Cancer Imaging 2018, 18, 52. [CrossRef]

78. Tu, W.; Sun, G.; Fan, L.; Wang, Y.; Xia, Y.; Guan, Y.; Li, Q.; Zhang, D.; Liu, S.; Li, Z. Radiomics signature: A potential and incremental predictor for EGFR mutation status in NSCLC patients, comparison with CT morphology. Lung Cancer 2019, 132, 28-35. [CrossRef]

79. Yang, X.; Dong, X.; Wang, J.; Li, W.; Gu, Z.; Gao, D.; Zhong, N.; Guan, Y. Computed Tomography-Based Radiomics Signature: A Potential Indicator of Epidermal Growth Factor Receptor Mutation in Pulmonary Adenocarcinoma Appearing as a Subsolid Nodule. Oncologist 2019, 24, e1156-e1164. [CrossRef]

80. Zhang, L.; Chen, B.; Liu, X.; Song, J.; Fang, M.; Hu, C.; Dong, D.; Li, W.; Tian, J. Quantitative Biomarkers for Prediction of Epidermal Growth Factor Receptor Mutation in Non-Small Cell Lung Cancer. Transl. Oncol. 2018, 11, 94-101. [CrossRef] [PubMed]

81. Zhao, W.; Wu, Y.; Xu, Y.; Sun, Y.; Gao, P.; Tan, M.; Ma, W.; Li, C.; Jin, L.; Hua, Y.; et al. The Potential of Radiomics Nomogram in Non-invasively Prediction of Epidermal Growth Factor Receptor Mutation Status and Subtypes in Lung Adenocarcinoma. Front. Oncol. 2019, 9, 1485. [CrossRef] [PubMed]

82. Zhao, W.; Yang, J.; Ni, B.; Bi, D.; Sun, Y.; Xu, M.; Zhu, X.; Li, C.; Jin, L.; Gao, P.; et al. Toward automatic prediction of EGFR mutation status in pulmonary adenocarcinoma with 3D deep learning. Cancer Med. 2019, 8, 3532-3543. [CrossRef] [PubMed]

83. Velazquez, E.R.; Parmar, C.; Liu, Y.; Coroller, T.P.; Cruz, G.; Stringfield, O.; Ye, Z.; Makrigiorgos, M.; Fennessy, F.; Mak, R.H.; et al. Somatic Mutations Drive Distinct Imaging Phenotypes in Lung Cancer. Cancer Res. 2017, 77, 3922-3930. [CrossRef] [PubMed]

84. Wang, X.; Kong, C.; Xu, W.; Yang, S.; Shi, D.; Zhang, J.; Du, M.; Wang, S.; Bai, Y.; Zhang, T.; et al. Decoding tumor mutation burden and driver mutations in early stage lung adenocarcinoma using CT-based radiomics signature. Thorac. Cancer 2019, 10, 1904-1912. [CrossRef] [PubMed]

85. Hong, D.; Xu, K.; Zhang, L.; Wan, X.; Guo, Y. Radiomics Signature as a Predictive Factor for EGFR Mutations in Advanced Lung Adenocarcinoma. Front. Oncol. 2020, 10, 28. [CrossRef]

86. Liu, Y.; Kim, J.; Balagurunathan, Y.; Li, Q.; Garcia, A.L.; Stringfield, O.; Ye, Z.; Gillies, R.J. Radiomic Features Are Associated with EGFR Mutation Status in Lung Adenocarcinomas. Clin. Lung Cancer 2016, 17, 441-448.e6. [CrossRef] [PubMed]

87. Song, L.; Zhu, Z.; Mao, L.; Li, X.; Han, W.; Du, H.; Wu, H.; Song, W.; Jin, Z. Clinical, Conventional CT and Radiomic Feature-Based Machine Learning Models for Predicting ALK Rearrangement Status in Lung Adenocarcinoma Patients. Front. Oncol. 2020, 10, 369. [CrossRef] [PubMed]

88. Sun, Z.; Hu, S.; Ge, Y.; Wang, J.; Duan, S.; Song, J.; Hu, C.; Li, Y. Radiomics study for predicting the expression of PD-L1 in non-small cell lung cancer based on CT images and clinicopathologic features. J. X-Ray Sci. Technol. 2020, 28, 449-459. [CrossRef] [PubMed]

89. Yoon, J.; Suh, Y.J.; Han, K.; Cho, H.; Lee, H.-J.; Hur, J.; Choi, B.W. Utility of CT radiomics for prediction of PD-L1 expression in advanced lung adenocarcinomas. Thorac. Cancer 2020, 11, 993-1004. [CrossRef] [PubMed]

90. Zhou, B.; Xu, J.; Tian, Y.; Yuan, S.; Li, X. Correlation between radiomic features based on contrast-enhanced computed tomography images and Ki-67 proliferation index in lung cancer: A preliminary study. Thorac. Cancer 2018, 9, 1235-1240. [CrossRef]

91. Gu, Q.; Feng, Z.; Liang, Q.; Li, M.; Deng, J.; Ma, M.; Wang, W.; Liu, J.; Liu, P.; Rong, P. Machine learning-based radiomics strategy for prediction of cell proliferation in non-small cell lung cancer. Eur. J. Radiol. 2019, 118, 32-37. [CrossRef]

92. Jiang, M.; Zhang, Y.; Xu, J.; Ji, M.; Guo, Y.; Guo, Y.; Xiao, J.; Yao, X.; Shi, H.; Zeng, M. Assessing EGFR gene mutation status in non-small cell lung cancer with imaging features from PET/CT. Nucl. Med. Commun. 2019, 40, 842-849. [CrossRef]

93. Koyasu, S.; Nishio, M.; Isoda, H.; Nakamoto, Y.; Togashi, K. Usefulness of gradient tree boosting for predicting histological subtype and EGFR mutation status of non-small cell lung cancer on 18F FDG-PET/CT. Ann. Nucl. Med. 2020, 34, 49-57. [CrossRef]

94. Li, X.; Yin, G.; Zhang, Y.; Dai, D.; Liu, J.; Chen, P.; Zhu, L.; Ma, W.; Xu, W. Predictive Power of a Radiomic Signature Based on 18F-FDG PET/CT Images for EGFR Mutational Status in NSCLC. Front. Oncol. 2019, 9, 1062. [CrossRef] 
95. Nair, J.K.R.; Saeed, U.A.; McDougall, C.C.; Sabri, A.; Kovacina, B.; Raidu, B.V.S.; Khokhar, R.A.; Probst, S.; Hirsh, V.; Chankowsky, J.; et al. Radiogenomic Models Using Machine Learning Techniques to Predict EGFR Mutations in Non-Small Cell Lung Cancer. Can. Assoc. Radiol. J. 2020, 72, 109-119. [CrossRef]

96. Zhang, J.; Zhao, X.; Zhao, Y.; Zhang, J.; Zhang, Z.; Wang, J.; Wang, Y.; Dai, M.; Han, J. Value of pre-therapy 18F-FDG PET/CT radiomics in predicting EGFR mutation status in patients with non-small cell lung cancer. Eur. J. Nucl. Med. Mol. Imaging 2020, 47, 1137-1146. [CrossRef]

97. Shiri, I.; Maleki, H.; Hajianfar, G.; Abdollahi, H.; Ashrafinia, S.; Hatt, M.; Zaidi, H.; Oveisi, M.; Rahmim, A. Next-Generation Radiogenomics Sequencing for Prediction of EGFR and KRAS Mutation Status in NSCLC Patients Using Multimodal Imaging and Machine Learning Algorithms. Mol. Imaging Biol. 2020, 22, 1132-1148. [CrossRef] [PubMed]

98. Yoon, H.J.; Sohn, I.; Cho, J.H.; Lee, H.Y.; Kim, J.-H.; Choi, Y.-L.; Kim, H.; Lee, G.; Lee, K.S.; Kim, J. Decoding Tumor Phenotypes for ALK, ROS1, and RET Fusions in Lung Adenocarcinoma Using a Radiomics Approach. Medicine 2015, 94, e1753. [CrossRef] [PubMed]

99. Jiang, M.; Sun, D.; Guo, Y.; Guo, Y.; Xiao, J.; Wang, L.; Yao, X. Assessing PD-L1 Expression Level by Radiomic Features from PET/CT in Nonsmall Cell Lung Cancer Patients: An Initial Result. Acad. Radiol. 2020, 27, 171-179. [CrossRef] [PubMed]

100. Yip, S.S.; Kim, J.; Coroller, T.P.; Parmar, C.; Velazquez, E.R.; Huynh, E.; Mak, R.H.; Aerts, H.J. Associations Between Somatic Mutations and Metabolic Imaging Phenotypes in Non-Small Cell Lung Cancer. J. Nucl. Med. 2017, 58, 569-576. [CrossRef] [PubMed]

101. Yip, S.S.; Parmar, C.; Kim, J.; Huynh, E.; Mak, R.H.; Aerts, H.J. Impact of experimental design on PET radiomics in predicting somatic mutation status. Eur. J. Radiol. 2017, 97, 8-15. [CrossRef] [PubMed]

102. Chen, B.T.; Jin, T.; Ye, N.; Mambetsariev, I.; Daniel, E.; Wang, T.; Wong, C.W.; Rockne, R.C.; Colen, R.; Holodny, A.I.; et al. Radiomic prediction of mutation status based on MR imaging of lung cancer brain metastases. Magn. Reson. Imaging 2020, 69, 49-56. [CrossRef] [PubMed]

103. Li, Y.; Cheng, Z.; Gevaert, O.; He, L.; Huang, Y.; Chen, X.; Huang, X.; Wu, X.; Zhang, W.; Dong, M.; et al. A CT-based radiomics nomogram for prediction of human epidermal growth factor receptor 2 status in patients with gastric cancer. Chin. J. Cancer Res. 2020, 32, 62-71. [CrossRef]

104. Zhang, Q.; Gao, Y.; Zhang, R.; Zhou, X.; Chen, S.; Zhang, Y.; Liu, Q.; Xu, J.; Ge, Z. Personalized CT-based radiomics nomogram preoperative predicting Ki-67 expression in gastrointestinal stromal tumors: A multicenter development and validation cohort. Clin. Transl. Med. 2020, 9, 12. [CrossRef]

105. Liang, W.; Yang, P.; Huang, R.; Xu, L.; Wang, J.; Liu, W.; Zhang, L.; Wan, D.; Huang, Q.; Lu, Y.; et al. A Combined Nomogram Model to Preoperatively Predict Histologic Grade in Pancreatic Neuroendocrine Tumors. Clin. Cancer Res. 2019, 25, 584-594. [CrossRef]

106. Yang, L.; Dong, D.; Fang, M.; Zhu, Y.; Zang, Y.; Liu, Z.; Zhang, H.; Ying, J.; Zhao, X.; Tian, J. Can CT-based radiomics signature predict KRAS/NRAS/BRAF mutations in colorectal cancer? Eur. Radiol. 2018, 28, 2058-2067. [CrossRef] [PubMed]

107. Wu, X.; Li, Y.; Chen, X.; Huang, Y.; He, L.; Zhao, K.; Huang, X.; Zhang, W.; Huang, Y.; Li, Y.; et al. Deep Learning Features Improve the Performance of a Radiomics Signature for Predicting KRAS Status in Patients with Colorectal Cancer. Acad. Radiol. 2020, 27, e254-e262. [CrossRef] [PubMed]

108. Lim, C.H.; Cho, Y.S.; Choi, J.Y.; Lee, K.-H.; Lee, J.K.; Min, J.H.; Hyun, S.H. Imaging phenotype using 18F-fluorodeoxyglucose positron emission tomography-based radiomics and genetic alterations of pancreatic ductal adenocarcinoma. Eur. J. Nucl. Med. Mol. Imaging 2020, 47, 2113-2122. [CrossRef] [PubMed]

109. Chen, S.-W.; Shen, W.-C.; Chen, W.T.-L.; Hsieh, T.-C.; Yen, K.-Y.; Chang, J.-G.; Kao, C.-H. Metabolic Imaging Phenotype Using Radiomics of [18F]FDG PET/CT Associated with Genetic Alterations of Colorectal Cancer. Mol. Imaging Biol. 2019, 21, 183-190 [CrossRef] [PubMed]

110. Cui, Y.; Liu, H.; Ren, J.; Du, X.; Xin, L.; Li, D.; Yang, X.; Wang, D. Development and validation of a MRI-based radiomics signature for prediction of KRAS mutation in rectal cancer. Eur. Radiol. 2020, 30, 1948-1958. [CrossRef]

111. Oh, J.E.; Kim, M.J.; Lee, J.; Hur, B.Y.; Kim, B.; Kim, D.Y.; Baek, J.Y.; Chang, H.J.; Park, S.C.; Oh, J.H.; et al. Magnetic ResonanceBased Texture Analysis Differentiating KRAS Mutation Status in Rectal Cancer. Cancer Res. Treat. 2020, 52, 51-59. [CrossRef]

112. Meng, X.; Xia, W.; Xie, P.; Zhang, R.; Li, W.; Wang, M.; Xiong, F.; Liu, Y.; Fan, X.; Xie, Y.; et al. Preoperative radiomic signature based on multiparametric magnetic resonance imaging for noninvasive evaluation of biological characteristics in rectal cancer. Eur. Radiol. 2019, 29, 3200-3209. [CrossRef]

113. Hectors, S.J.; Lewis, S.; Besa, C.; King, M.J.; Said, D.; Putra, J.; Ward, S.; Higashi, T.; Thung, S.; Yao, S.; et al. MRI radiomics features predict immuno-oncological characteristics of hepatocellular carcinoma. Eur. Radiol. 2020, 30, 3759-3769. [CrossRef]

114. Ye, Z.; Jiang, H.; Chen, J.; Liu, X.; Wei, Y.; Xia, C.; Duan, T.; Cao, L.; Zhang, Z.; Song, B. Texture analysis on gadoxetic acid enhanced-MRI for predicting Ki-67 status in hepatocellular carcinoma: A prospective study. Chin. J. Cancer Res. 2019, 31, 806-817. [CrossRef]

115. Yao, Z.; Dong, Y.; Wu, G.; Zhang, Q.; Yang, D.; Yu, J.-H.; Wang, W.-P. Preoperative diagnosis and prediction of hepatocellular carcinoma: Radiomics analysis based on multi-modal ultrasound images. BMC Cancer 2018, 18, 1089. [CrossRef]

116. Peng, Y.-T.; Zhou, C.-Y.; Lin, P.; Wen, D.-Y.; Wang, X.-D.; Zhong, X.-Z.; Pan, D.-H.; Que, Q.; Li, X.; Chen, L.; et al. Preoperative Ultrasound Radiomics Signatures for Noninvasive Evaluation of Biological Characteristics of Intrahepatic Cholangiocarcinoma. Acad. Radiol. 2019, 27, 785-797. [CrossRef] 
117. Saadani, H.; Van Der Hiel, B.; Aalbersberg, E.A.; Zavrakidis, I.; Haanen, J.B.; Hoekstra, O.S.; Boellaard, R.; Stokkel, M.P. Metabolic Biomarker-Based BRAFV600 Mutation Association and Prediction in Melanoma. J. Nucl. Med. 2019, 60, 1545-1552. [CrossRef]

118. Yoon, J.H.; Han, K.; Lee, E.; Lee, J.; Kim, E.-K.; Moon, H.J.; Park, V.; Nam, K.-H.; Kwak, J.Y. Radiomics in predicting mutation status for thyroid cancer: A preliminary study using radiomics features for predicting BRAFV600E mutations in papillary thyroid carcinoma. PLoS ONE 2020, 15, e0228968. [CrossRef]

119. Zhu, Y.; Mohamed, A.S.; Lai, S.; Yang, S.; Kanwar, A.; Wei, L.; Kamal, M.; Sengupta, S.; ElHalawani, H.; Skinner, H.; et al. Imaging-Genomic Study of Head and Neck Squamous Cell Carcinoma: Associations Between Radiomic Phenotypes and Genomic Mechanisms via Integration of The Cancer Genome Atlas and The Cancer Imaging Archive. JCO Clin. Cancer Inform. 2019, 3, 1-9. [CrossRef] [PubMed]

120. Chen, R.-Y.; Lin, Y.-C.; Shen, W.-C.; Hsieh, T.-C.; Yen, K.-Y.; Chen, S.-W.; Kao, C.-H. Associations of Tumor PD-1 Ligands, Immunohistochemical Studies, and Textural Features in 18F-FDG PET in Squamous Cell Carcinoma of the Head and Neck. Sci. Rep. 2018, 8, 105. [CrossRef]

121. Ahmed, A.; Elmohr, M.; Fuentes, D.; Habra, M.; Fisher, S.; Perrier, N.; Zhang, M.; Elsayes, K. Radiomic mapping model for prediction of Ki-67 expression in adrenocortical carcinoma. Clin. Radiol. 2020, 75, 479.e17-479.e22. [CrossRef] [PubMed]

122. Bogowicz, M.; Jochems, A.; Deist, T.M.; Tanadini-Lang, S.; Huang, S.H.; Chan, B.; Waldron, J.N.; Bratman, S.; O'Sullivan, B.; Riesterer, O.; et al. Privacy-preserving distributed learning of radiomics to predict overall survival and HPV status in head and neck cancer. Sci. Rep. 2020, 10, 1-10. [CrossRef] [PubMed]

123. Shi, Z.; Zhovannik, I.; Traverso, A.; Dankers, F.J.W.M.; Deist, T.M.; Kalendralis, P.; Monshouwer, R.; Bussink, J.; Fijten, R.; Aerts, H.J.W.L.; et al. Distributed radiomics as a signature validation study using the Personal Health Train infrastructure. Sci. Data 2019, 6, 1-8. [CrossRef] [PubMed]

124. Bogowicz, M.; Vuong, D.; Huellner, M.W.; Pavic, M.; Andratschke, N.; Gabrys, H.S.; Guckenberger, M.; Tanadini-Lang, S. CT radiomics and PET radiomics: Ready for clinical implementation? Q. J. Nucl. Med. Mol. Imaging 2019, 63, 355-370. [CrossRef] [PubMed]

125. Lee, J.; Steinmann, A.; Ding, Y.; Lee, H.; Owens, C.; Wang, J.; Yang, J.; Followill, D.; Ger, R.; MacKin, D.; et al. Radiomics feature robustness as measured using an MRI phantom. Sci. Rep. 2021, 11, 1-14. [CrossRef]

126. Van Timmeren, J.E.; Cester, D.; Tanadini-Lang, S.; Alkadhi, H.; Baessler, B. Radiomics in medical imaging- “How-to” guide and critical reflection. Insights Imaging 2020, 11, 91. [CrossRef] [PubMed]

127. The Image Biomarker Standardisation Initiative-IBSI 0.0.1dev Documentation. Available online: https://ibsi.readthedocs.io/ en/latest/ (accessed on 17 November 2020).

128. Aronson, J.K.; Ferner, R. Biomarkers-A General Review. Curr. Protoc. Pharmacol. 2017, 76, 9.23.1-9.23.17. [CrossRef] [PubMed] 